\title{
Moyens de subsistance des adolescentes. Questions primordiales et outils essentiels : Rapport d'un atelier
}

Carey Meyers

Population Council

Follow this and additional works at: https://knowledgecommons.popcouncil.org/departments_sbsr-pgy

Part of the Demography, Population, and Ecology Commons, Family, Life Course, and Society Commons, International Public Health Commons, and the Labor Economics Commons How does access to this work benefit you? Let us know!

\section{Recommended Citation}

Meyers, Carey. 2004. "Moyens de subsistance des adolescentes. Questions primordiales et outils essentiels : Rapport d'un atelier." New York and Washington, DC: Population Council and International Center for Research on Women. 

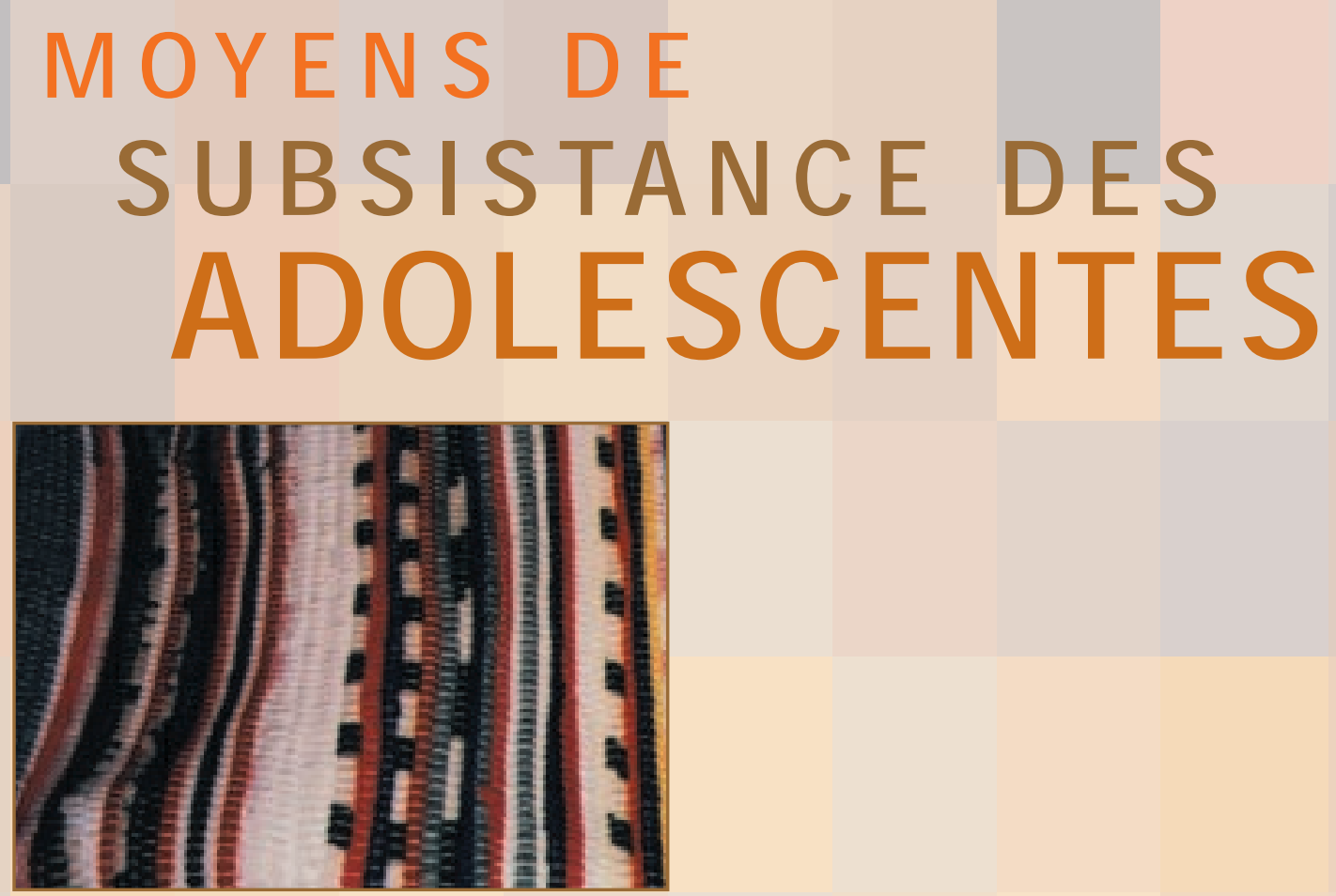

QUESTIONS PRIMORDIALES ET OUTILS ESSENTIELS : RAPPORT D'UN ATELIER

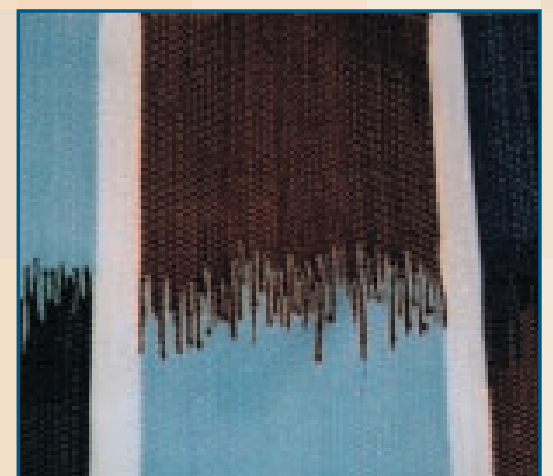


L'art qui se trouve à la couverture doit être familier aux participants de l'atelier « Questions primordiales et outils essentiels » ou à ceux qui connaissent l'Association pour la protection de l'environnement (APE), une ONG égyptienne. Les photos sur la couverture ainsi que celles qui se trouvent partout dans le rapport sont des détails des motifs traditionaux égyptiens qui sont entrelacés dans le tissage des adolescentes qui produisent des sacs et des tapis à l'APE. L'APE dirige des programmes novateurs de subsistance pour les adolescentes non-mariées qui vivent à Maqattam, une communauté pauvre, traditionnelle, et marginalisée dans la banlieu du Caire. L'APE a organisé une visite sur le terrain pour les participants de l'atelier, et a fourni un sac à chacun. Les adolescentes ont aussi exhibé leurs marchandises pendant l'atelier.

Les détails sur le projet de l'APE se trouvent dans la 19ème édition de SEEDS, une série de publications du Population Council qui documente des programmes qui aident aux femmes à gagner leur vie et à améliorer leur statut économique. Des copies de SEEDS peuvent être téléchargées du site web du Population Council, www.popcouncil.org. Pour une copie imprimée, veuillez contacter le Population Council à l'adresse ci-dessous.

\section{Population Council}

One Dag Hammarskjold Plaza

New York, New York 10017

téléphone: 001 212-339-0500

fax: 001 212-755-6052

e-mail: pubinfo@popcouncil.org

www.popcouncil.org

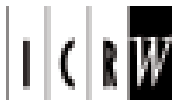

1717 Massachusetts Avenue NW, Suite 302

Washington, DC 20036

téléphone: 001 202-797-0007

fax: 001 202-797-0020

e-mail:info@icrw.org

www.icrw.org

Population Council est une organisation internationale, non gouvernementale, à but non lucratif qui cherche à améliorer le bien-être et la santé de la reproduction des générations actuelles et futures à travers le monde et à aider à l'établissement d'un équilibre humain équitable et durable entre les populations et les ressources. Population Council effectue des recherches dans les domaines de la biomédecine, des sciences sociales et de la santé publique et aide à renforcer les outils de recherche dans les pays en voie de développement. Créé en 1952, le Council est dirigé par un Conseil d'administration dont les membres sont originaires de différents pays. Son siège à New York apporte un soutien à tout un réseau de bureaux nationaux et régionaux.

L'International Center for Research on Women (ICRW) est une organisation privée à but non lucratif qui se consacre à la promotion du développement axé sur une pleine participation de la femme. L'ICRW oriente son action sur la recherche en matière des et à l'appui aux programmes sur les femmes et leurs rôles productifs et reproductifs, leur statut dans la famille, leur direction dans la sociéte, et leur rôle dans la protection des ressources environnementales. L'ICRW travaille en collaboration avec des responsables des gouvernements et des agences multilatérales, convoque des experts dans des réunions formelles et informelles, et s'occupe d'un programme actif de communication et publication pour promouvoir les droits ainsi que les opportunités des filles et femmes du monde. Créée en 1976, l'ICRW s'oriente principalement vers les femmes dans les pays en développement et en transition.

\section{(C) 2004 Population Council et International Center for Research on Women}

Toute partie de ce document peut être reproduite ou adaptée aux besoins locaux sans la permission du Population Council ni de l'ICRW, pourvu qu'elle soit distribuée gratuitement ou à des fins non lucratives et que la source soit identifiée. Toute reproduction commerciale nécessite la permission à l'avance du Population Council ou de l'ICRW. Le Population Council et l'International Center for Research on Women voudraient bien recevoir une copie de tous les matériaux qui emploient le texte. 


\section{TABLE DES MATIÈRES}

REMERCIEMENTS $\mathrm{V}$

POURQUOI ÉTUDIER LES MOYENS DE SUBSISTANCE DES ADOLESCENTS ? 1

\begin{tabular}{ll}
\hline CARACTÉRISTIQUES DU PRÉSENT RAPPORT & 3
\end{tabular}

$\begin{array}{ll}\text { QUESTIONS PRIMORDIALES } & 4\end{array}$

Où travaillent les adolescents?

Insuffisances des données sur la main d'œuvre? $\quad 8$

Celles qui ne travaillent pas et qui ne fréquentent pas l'école

« ne font-elles vraiment rien »? 9

LE TRAVAIL DES FILLES ET L'ENVIRONNEMENT POLITIQUE ET NORMATIF $\quad 16$

Quelles sont les contradictions entre le travail et l'éducation? 16

Promotion de milieux de travail sûrs et productifs pour les adolescentes:

normes de I'UNICEF et du BIT et l'industrie de la confection

au Bangladesh

COMMENT LES JEUNES ADOLESCENTES PERÇOIVENT-ELLES LEURS

CONDITIONS DE TRAVAIL?

COMMENT LE TRAVAIL CONTRIBUE-T-IL À LEUR « ADOLESCENCE »?

POINT DE VUE DE TROIS PAYS 22

Égypte $\quad 22$

La Jordanie $\quad 25$

$\begin{array}{ll}\text { Le Bangladesh } & 28\end{array}$

FONDEMENTS DES CHOIX POLITIQUES ET PROGRAMMES DESTINÉS

À ASSURER DES MOYENS DE SUBSISTANCE SÛRS ET APPROPRIÉS

$\begin{array}{ll}\text { AUX ADOLESCENTES PLUS ÂGÉES } & 31\end{array}$

Comment trouver le cadre théorique de la politique adéquate? 31

À ce jour quelle est l'expérience en matière du création et/ou

de soutien des moyens de subsistance? 38

Quelles sont les possibilités d'implication des adolescents dans

les programmes de microfinance? Dans quelles circonstances

cela peut-il se faire correctement?

Quels sont les éléments d'une formation appropriée en matière de création d'entreprise?

QUE RÉSERVE L'AVENIR?

NOTES

RÉFÉRENCES

ANNEXE A: L'ORDRE DU JOUR DE L'ATELIER

ANNEXE B: LISTE DE PARTICIPANTS DE L'ATELIER 


\section{REMERCIEMENTS}

Questions primordiales et outils essentiels, tel est le thème d'un atelier qui s'est tenu du 13 au 14 octobre 1999 au Caire, en Égypte et qui se rapporte à un effort de planification conjointement mené par les bureaux du Population Council de New York et du Caire et le Centre international pour la recherche sur les femmes (ICRW). Aux moments décisifs, les deux organisations ont considérablement tiré parti des consultations avec leurs collègues du Fonds des Nations Unies pour l'Enfance (UNICEF). La Fondation des Nations Unies a fourni le financement de la réunion et grâce à cette dotation, joué un rôle déterminant en favorisant la prise de conscience sur les moyens de subsistance des adolescents.

Le Centre de recherche pour le développement international (CRDI) et notamment Jamie Schnurr et Necla Tchirgi, continuent d'animer un débat intellectuel courageux axé sur les moyens de subsistance des adolescents. L'organisation a contribué au financement de cet atelier et a mis en rapport les organisateurs avec les individus et les associations engagés dans des initiatives novatrices qui pourraient faire l'objet d'études de cas durant les travaux de l'atelier (y compris le Réseau du CRDI des moyens de subsistance en Afrique), basé à l'université de Venda en Afrique du Sud, dont la présence a été particulièrement appréciée).

Nous exprimons également notre reconnaissance à l'organisation caritative William H. Kaufman et à Effie Westervelt pour leur appui financier dans le cadre de la réunion et à la Fondation Ford pour l'assistance qu'elle a apportée à la publication du présent rapport.

En sa qualité de rapporteur de la réunion, Carey Meyers a rédigé toutes les synthèses, élaboré le rapport et supervisé sa production. Cependant, le produit final a bénéficié de la collaboration de nombre de personnes, à savoir Susan Lee pour la première révision ; en leur qualité d'examinateurs, Jamie Schnurr, Simel Esim, Judith Bruce, Jennefer Sebstad, Bruce Dick et Geeta Rao Gupta ont de manière perspicace formulé des observations et fourni des commentaires écrits en temps opportun ; Nicole Haberland et Rachel Goldberg ont, tout au long du processus de production, apporté leur assistance technique. 


\section{POURQUOI ÉTUDIER LES MOYENS DE SUBSISTANCE DES ADOLESCENTS?}

Les mesures d'assistance aux adolescents — considérés le plus souvent comme étant le groupe d'âge des 10 à 19 ans recoupent aussi bien les initiatives destinées à l'enfance que celles destinées à la jeunesse. Jusque récemment, les efforts se sont dans la majorité des cas, résumés à assurer l'éducation, un milieu environnant sûr, une nutrition adéquate ainsi que des informations relatives à la santé et des services y afférents. La Convention des Nations Unies sur les Droits de l'Enfant, adoptée en 1989, et qui fixe l'âge maximal de l'enfance à 18 ans, a donné une importance croissante aux droits des adolescents. Cependant, dans un environnement où les initiatives étaient déjà réduites, l'on ne s'est pas beaucoup préoccupé d'appréhender la différence entre les besoins des filles et ceux des garçons, de comprendre les expériences professionnelles des adolescents plus jeunes et plus âgés et de renforcer les capacités de leurs moyens de subsistance. Le sujet portant sur les moyens de subsistance des adolescents s'est également heurté au manque de données appréciables. Il est primordial de distinguer l'expérience et les droits des adolescents plus âgés de ceux plus jeunes, leurs emplois et leurs situations relatives et respectives sur le marché du travail. Les politiques, les chercheurs, et les planificateurs doivent admettre que les adolescents âgés de 10 à 14 ans ont des besoins différents et sont protégés par un ensemble différent de lois par rapport aux jeunes âgés de 15 ans ou plus, qui peuvent dans la majorité des cas, faire partie de la population active.

De manière générale, les adolescents participent à la vie active parce qu'ils éprouvent le besoin économique de contribuer à réduire la vulnérabilité de leur famille : les familles envoient les adolescents travailler dans le cadre d'une stratégie plus globale de survie du ménage. L'intérêt qu'une famille peut manifester pour le travail d'un adolescent masque parfois les avantages potentiels que ceux-ci peuvent tirer de leur emploi et de leur droit à développer leur capacité à gagner leur vie. Bien que les adolescents ne soient pas toujours à l'origine de leur propre participation à la vie active, ils se voient offrir des possibilités d'apprendre, de grandir en tant qu'individus et d'entrevoir ce qu'ils aimeraient que l'avenir leur réserve. En somme, le moment où une jeune personne participe à la vie active et la manière dont elle s'y prend peuvent déterminer son futur statut et les opportunités d'emploi qu'elle aura. Pour les adolescents et adolescentes, les moyens de subsistance sont les fondements de leur futur bien-être. Pour les filles et les femmes notamment, le pouvoir de négociation dans la vie conjugale et en matière de fécondité, demeurera limité si elles ne sont pas en mesure de gagner leur vie de manière indépendante. 


\section{RECONNAISSANCE DES BESOINS SPÉCIFIQUES DES FILLES}

epuis 1997, les estimations du Bureau international du travail (BIT) indiquent que 110 millions de filles travaillent à travers le monde, contre 140 millions de garçons. Bien que les filles puissent travailler à côté des garçons dans presque tous les secteurs, les autres travaux effectués par les filles, tels que les travaux domestiques rémunérés ou non, sont souvent imperceptibles, ce qui contribue à dénombrer de manière inexacte le nombre de filles qui travaillent. De plus, les filles sont peutêtre beaucoup plus vulnérables au traitement inéquitable sur le marché du travail, car l'insertion sociale par sexe leur apprend à être dociles et obéissantes dès le plus jeune âge, attitude qui a aussi un impact sur leurs stratégies de survie. Des filles qui ont peu d'opportunités sûres et productives peuvent devenir à l'âge adulte, des femmes qui travaillent avec peu d'options sûres et productives. De même, les jeunes filles ont moins d'opportunités que les garçons d'avoir un emploi rémunéré hors de la maison, étant donné qu'elles sont souvent chargées de s'occuper de la maison pendant que leur mère va au travail. Les filles qui travaillent hors de leur domicile sont pourtant souvent chargées d'une part importante des travaux domestiques et elles ploient en réalité sous deux types de travaux.

Au niveau communautaire, il est crucial de développer et de donner libre cours à l'énergie des jeunes, notamment dans les communautés pauvres où les garçons ainsi que les filles, seront bientôt chargés non seulement de subvenir à leurs propres besoins mais également à ceux de leur famille. La mondialisation de l'économie offre aux adolescents plus âgés, particulièrement les filles, des possibilités sans précédent et cependant potentiellement incomprises de gagner des revenus qui peuvent accroître leur statut social et économique, leur amour-propre et leurs compétences. Dans certains pays dont les données sont disponibles, il apparaît que les jeunes femmes ont la mainmise sur nombre de nouvelles industries axées sur les exportations. Des préoccupations relatives à l'exploitation des adolescents et adolescentes qui travaillent dans les usines dans d'autres environnements, ont en grande partie, dominé le débat d'orientation de politique. Ces préoccupations tout à fait justifiées méritent l'attention requise, mais elles ne doivent pas occulter les avantages potentiels que ces opportunités croissantes de travail rémunéré, offrent non seulement aux filles en tant qu'individus mais également à la société. 
L'atelier «Questions primordiales et outils essentiels » a été motivé par le désir d'en apprendre davantage sur la nature de l'expérience des adolescents plus jeunes et plus âgés en matière d'emploi, de distinguer les besoins particuliers et potentiels des adolescentes et d'identifier des programmes et politiques quels qu'en soient l'échelle et les modalités, qui leur offrent des perspectives de soutien. L'atelier s'est par conséquent attaché à répondre aux cinq questions primordiales qui suivent :
- Où travaillent les adolescents ?

- Quel est l'environnement politique et normatif qui régit l'emploi des filles?

- Comment les adolescentes font-elles face à leurs conditions de travail de quelle manière leur emploi contribue-t-il à leur adolescence?

- Quelle est la méthode adoptée pour gagner leur vie?

- À ce jour, quelles sont les initiatives réalisées en matière de soutien et/ou de génération de moyens de subsistance au profit des adolescentes?

\section{CARACTÉRISTIQUES DU PRÉSENT RAPPORT}

Le présent rapport ne respecte pas rigoureusement l'ordre du jour de la réunion parce qu'il tente de mettre en rapport la mine de renseignements présentés durant plus de deux jours au Caire de la manière la plus lisible possible. Alors qu'il repose sur les résumés individuels de chaque communication, les informations tirées de deux communications ou plus ont dans certains cas été combinées afin de faciliter la fluidité du texte. Des notes à la fin indiquent la communication du présentateur dont chaque section du rapport trouve sa source. L'ordre du jour, joint en Annexe A, fournit les noms de ceux qui ont présenté des communications et les lecteurs sont encouragés à contacter directement les présentateurs s'ils ont besoin d'informations supplémentaires sur un sujet particulier. Les coordonnées figurent en Annexe B.

Ce rapport tente de mettre en exergue le thème des "Questions primordiales et outils essentiels » en libellant son texte rédactionnel à partir des questions qui sont abordées ci-dessus. Une «panoplie » épisodique jette un éclairage sur les sujets de recherche qui doivent faire l'objet d'un examen. 


\section{QUESTIONS PRIMORDIALES ${ }^{1}$}

Nous savons qu'environ 250 millions d'adolescents et d'enfants âgés de 5 à 14 ans travaillent d'une manière ou d'une autre pour une rémunération et l'on estime à 120 millions le nombre de ceux qui travaillent à plein temps (BIT 1996). Selon les estimations les plus fiables, 61 pour cent du nombre total d'adolescents sont employés en Asie, 32 pour cent en Afrique, et 7 pour cent en Amérique Latine. En dépit de tous ces chiffres significatifs, très peu d'informations sont réellement disponibles sur la demande d'emploi émanant des adolescents et leur désir de travailler ou encore sur leur expérience en matière d'emploi. En fonction des informations facilement accessibles et déjà publiées, il est clair que la demande de travail existe et cependant l'origine de cette demande constitue l'un des multiples aspects de l'expérience des adolescents en matière d'emploi, qui nous échappent. Les autres questions primordiales se présentent comme suit :

- La demande d'emploi des adolescents est-elle suscitée par eux-mêmes ou par leurs parents ? Pour quelles raisons les adolescents travaillentils ? Le font-ils dans le but d'acquérir des compétences, dans le cadre de la stratégie de survie de la famille ou afin de gagner de l'argent en vue du mariage?

- Quelles sont les principales sources d'emploi pour les jeunes ? Combien d'entre eux travaillent dans des entre- prises familiales, des usines ou sont installés à leur propre compte?

- Quel est le rôle de la législation en matière de travail des enfants dans la détermination des opportunités d'emploi pour les adolescents?

- Dans quelle mesure les jeunes peuvent-ils bénéficier des mécanismes de microcrédit et des régimes d'épargne? Cette recevabilité varie-t-elle en fonction du statut matrimonial ?

- Quel type de compétences peut-on acquérir dans le cadre des programmes de service national ? ${ }^{2}$

- De quelle manière les adolescents passent-ils leur temps pendant une journée ordinaire?

- Comment les activités évoluent-elles les jours d'école par opposition aux jours sans école?

- Quelle est le prévalence de l'emploi des adolescents?

- Quels types d'emploi les adolescents occupent-ils ? Dans quelles conditions ? Où ? Comment sont-ils rémunérés ? Le sont-ils en espèces ou en nature ? S’agit-il de travail non rémunéré ? Acquièrent-ils des compétences ?

- Lorsque les adolescents gagnent effectivement de l'argent, qui contrôle leurs revenus ? Comment utilisent-ils ces revenus?

Il est très difficile de recueillir des données sur la manière dont les adolescents, particulièrement les jeunes adolescentes 
passent leur temps. Il est clair qu'une meilleure compréhension de la manière dont ils en font usage permettrait de déterminer considérablement les possibilités d'interventions programmatiques. Il est également important de comprendre les conditions de travail. Dans beaucoup de sociétés par exemple, les horaires scolaires sont, de manière surprenante, réduits ; la fréquentation scolaire n'est ainsi pas incompatible avec un emploi. Parmi ceux qui ont participé à l'activité économique et qui l'ont ensuite quittée, quelles sont les circonstances qui déterminent leur retour ultérieur sur le marché du travail ? Le fait d'évaluer le temps que les adolescents passent à l'école, le temps qu'ils consacrent aux travaux ménagers et familiaux et le temps qui leur reste pour les loisirs, nous fournit des informations sur leur rythme de vie. Des enquêtes portant sur la main d'œuvre donnent un aperçu de l'importance de l'emploi des adolescents, il est toutefois difficile d'interpréter sa véritable envergure.

\section{Où travaillent les adolescents ?}

Le Bureau international du travail (BIT) publie périodiquement des statistiques sur l'emploi, qui révèlent certaines caractéristiques de l'emploi des jeunes, notamment les taux de participation à la vie active selon l'âge. ${ }^{3}$ D’après ces données, nous savons que les taux d'activités économiques selon l'âge et le sexe varie d'un pays à l'autre (Graphiques 1-3).

Une répartition du pourcentage des garçons et des filles de moins de 15 ans,
GRAPHIQUE 1. Taux d'activité économique par groupe d'âge et sexe, Égypte

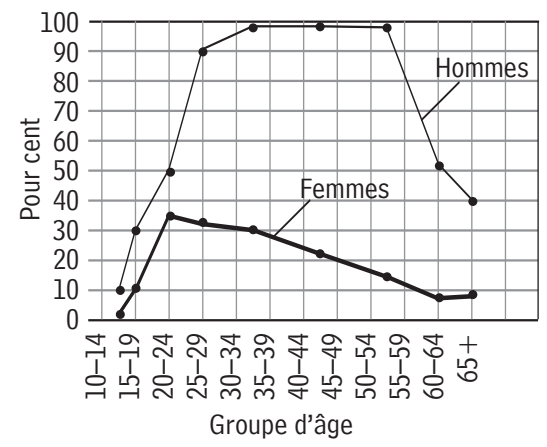

En Égypte, le taux global de participation est aussi faible pour les garçons que pour les filles - il existe un fossé des sexes mais il n'est pas important. Pour les filles, la participation à la vie active culmine à l'âge de 20 à 24 et elle baisse ensuite. Cela indique qu'il existe pour les femmes peu d'opportunités de travailler dans le secteur formel après le mariage.

Source : Bureau international du travail 1993, 1994.

économiquement actifs montre qu'ils travaillent dans trois secteurs principaux : I'agriculture (y compris la chasse, la forestèrie et la pêche) ; les services communautaires, sociaux et personnels ainsi que le secteur de la fabrication. L'agriculture, avec de loin la participation la plus importante, emploie près de 80 pour cent des filles et 75 pour cent des garçons. Si l'on inclut la participation nominale des garçons et des filles dans les secteurs de la fabrication et des services communautaires/sociaux/personnels, on obtient un tableau exhaustif de la participation des garçons et des filles de moins de 15 ans à la vie active.

Le BIT a récemment lancé une initiative spéciale destinée à améliorer l'évaluation de l'activité économique des enfants 
GRAPHIQUE 2. Taux d'activité économique par groupe d'âge et sexe, Nigéria

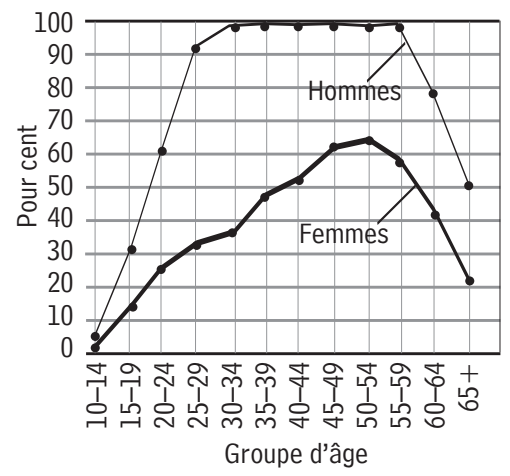

GRAPHIQUE 3. Taux d'activité économique par groupe d'âge et sexe, Thaïlande

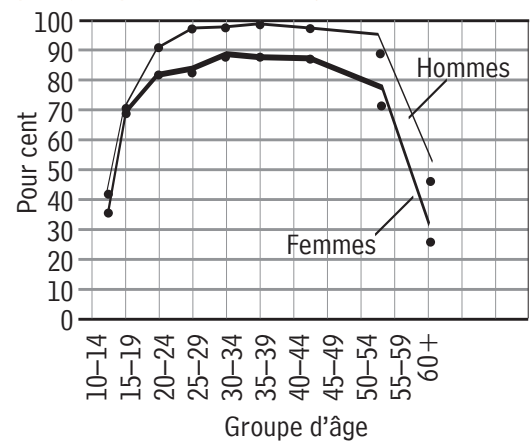

Au Nigéria, le profil de la participation à la vie active est semblable à celui de l'Égypte au cours de l'adolescence, mais les disparités entre les sexes s'amenuisent plutôt qu'elles ne s'accroissent avec le temps. En Thaïlande, le fossé des sexes est dérisoire durant l'adolescence. Il s'accroît chez les adultes mais de manière peu significative puisque les taux de participation chez les hommes et les femmes augmentent et baissent simultanément au cours de leur vie.

Source : Bureau international du travail 1993, 1994.

âgés de 5 à 14 ans. L'expérience a démarré au Ghana, en Inde, en Indonésie et au Sénégal en se basant sur une enquête des ménages. Il a été demandé aux sujets interrogés s'ils travaillent contre une rémunération en espèces ou en nature ou en qualité de travailleurs familiaux non rémunérés et des questions leur ont été posées sur leurs activités présentes et habituelles. Les ménages ont été classés en trois groupes : ceux dont au moins un enfant a un emploi rémunéré, ceux ayant au moins un enfant rémunéré et un non rémunéré et les autres. Pour les pays disposant d'autres données disponibles, des comparaisons ont montré des taux d'acti-vité économique beaucoup plus élevés que par le passé.

La principale conclusion de cette initiative était que la qualité des données sur l'emploi du temps était décevante. Il est apparu qu'au plus 12 pour cent des enfants âgés de 5 à 13 ans déclarent que travailler a été leur principale activité durant les sept jours précédents et l'on trouve même une plus grande participation lorsque la période est élargie. Sans perdre de vue les insuffisances des données, l'étude a aussi révélé que :

- Proportionnellement, plus de garçons que de filles prennent part à une activité économique ;

- Même si les filles ne sont pas en principe prises en compte dans les enquêtes sur la main d'œuvre, un nombre important d'entre elles participent à des activités économiques non rémunérées à domicile ;

- En milieu rural, les taux d'activité économique des enfants sont deux fois plus élevés que dans les zones urbaines, essentiellement en raison de leur participation aux travaux agricoles ;

- Parmi les enfants qui travaillent, presque tous sont dans le secteur informel et essentiellement dans les entreprises familiales; et 
- Les enfants qui travaillent comprennent les étudiants et les non étudiants ; les étudiants qui travaillent sont particulièrement absents des représentations classiques de la main d'œuvre.

Les adolescents, définis le plus souvent comme le groupe d'âge compris entre 10 et 19 ans, comprennent les enfants et les jeunes. La catégorie des jeunes comprend ceux qui sont âgés de 15 à 30 ans alors que celle des enfants peut aller jusqu'à 15 ans. La majeure partie des recherches sur le développement et des programmes axés sur les adolescents portent sur le comportement sexuel et procréateur (Mensch, Bruce et Greene 1998). Cependant, la participation des adolescents plus jeunes (10 à 14 ans) et plus âgés (15 à 19 ans) à la vie active apparaît comme un aspect important du développement. La convention No 138 du BIT indique 15 ans comme étant l'âge minimal auquel un individu peut commencer à participer à l'activité économique.

En tenant compte des insuffisances des données, il est important d'établir la distinction pour ce qui concerne l'expérience des adolescents plus âgés et plus jeunes en matière d'emploi ainsi que les postes qu'occupent les adolescents des deux sexes sur le marché de l'emploi. Il existe de fortes divergences sur les significations que l'on donne au travail des adolescents plus jeunes - qui selon l'avis le mieux partagé, travaillent illégalement — et à celui des adolescents plus âgés - qui dans la majorité des cas peuvent légitimement participer à la vie active.
Peu d'informations sont disponibles sur les motivations de l'emploi des adolescents, son importance et ses conditions. En apparence, la pauvreté constitue la principale raison qui sous-tend le travail des adolescents. Ils travaillent pour assurer leur survie et celle de leur famille. L'augmentation du nombre des membres de la famille qui travaillent, réduisant ainsi le risque en diversifiant les activités génératrices de revenus, peut constituer, soit une importante stratégie de survie en temps de difficultés, soit un moyen d'accroître les revenus du ménage et de lutter contre la pauvreté chronique (Szanton Blanc 1994). Ainsi les gains des adolescents qui travaillent peuvent être perçus comme un moyen important d'accroître les revenus du ménage et de réduire son instabilité. Dans une étude portant sur les enfants de la rue au Paraguay, 50 pour cent des enfants de la rue qui travaillent, ont contribué au revenu total du ménage d'au moins la moitié (Espínola et al. 1988). Le travail non rémunéré productif — pourtant vital est également assez commun chez les adolescentes qui s'occupent des tâches ménagères et des enfants afin de permettre à leur mère d'avoir le temps de se consacrer à un travail rémunéré. Les tâches incluent la garde d'enfants, la préparation des repas, la recherche de l'eau et de combustible, le ménage et les travaux agricoles (Mensch, Bruce et Greene 1988).

Le chômage des jeunes a plusieurs implications pour le marché de l'emploi, pour les ménages pauvres et pour les adolescents eux-mêmes. L'incapacité à trouver 
du travail renforce l'exclusion économique, la pauvreté et la probabilité d'un futur chômage. Le chômage empêche non seulement les jeunes de contribuer à l'économie de manière productive, mais des conséquences sanitaires et sociales pourraient également en découler (par exemple, l'isolement et l'incapacité à satisfaire les besoins nutritionnels). Étant donné que le travail est un moyen important qui permet aux jeunes de développer des rôles d'adultes et de responsabilité, le chômage compromet le passage des jeunes de l'adolescence à l'âge adulte.

\section{Insuffisances des données sur la main d'œuvre?}

Les insuffisances des données relatives à la vie active pour les adolescents sont analogues à celles qui s'appliquent aux adultes, notamment aux femmes. Avec les adolescents, les insuffisances sont exacerbées parce que les taux de participation peuvent considérablement varier d'une semaine à l'autre, d'un mois à l'autre ou à cause des activités scolaires. Par exemple, les enquêtes sur la main d'œuvre incluent toujours une période de référence, habituellement « la semaine précédente », lorsque des questions sont posées sur l'activité principale du sujet interrogé. La réponse d'un adolescent sera différente parmi ceux qui fréquentent l'école, certains considéreront leur activité principale comme se limitant aux « études » alors que d'autres considéreront que la leur concerne le « travail ». Il en résulte que la participation des adolescents à la main d'œuvre peut être sous-estimée. De même, les a recherche de données utiles à

partir de diverses sources existantes permet de déterminer un profil plus nuancé des adolescents au travail. Ces sources peuvent inclure :

- Des enquêtes sur la main d'œuvre familiale

- Des études sur l'emploi du temps

- Des enquêtes démographiques et sanitaires ;

- Des enquêtes sur la mesure des niveaux de vie ; et

- Des enquêtes portant sur le secteur informel.

Plusieurs pays disposent de très bonnes sources de données qui peuvent fournir des informations transversales sur la vie des adolescentes. Il nous reste beaucoup à apprendre de l'analyse des données secondaires. Une collaboration entre les différentes disciplines - l'économie, la sociologie et la démographie permet souvent d'avoir une meilleure perception de ce qui se passe réellement dans la vie active.

enquêtes sur la main d'œuvre insistent fortement sur les emplois rémunérés, alors que la définition que l'on donne de la rémunération est peu satisfaisante. Des différences relatives à l'âge civil pour être éligible à l'emploi et les différents niveaux d'application de la législation y afférente, jettent un voile sur la compréhension que nous en avons bien que cela devienne plus 
clair à la fin de l'adolescence. Il n'est pas aisé de tirer des conclusions sur les différences entre les sexes chez les adolescents en raison de leur sous-estimation. En réalité, il se peut que les fossés des sexes soient moins importants que ceux dont il est fait état parmi les adolescents qui travaillent en raison d'une déclaration inadéquate et de leur non prise en compte dans les enquêtes.

\section{Celles qui ne travaillent pas et qui ne fréquentent pas l'école « ne font-elles vraiment rien "?}

L'absence de corrélation entre la participation des jeunes filles à la vie active et leur fréquentation scolaire donnent aux chercheurs une idée confuse de la manière dont les adolescentes passent leur temps. Même si l'on tient compte du manque de données de qualité sur les activités professionnelles des filles, une importante fraction d'entre elles dans les pays en développement ne travaillent pas, ne fréquentent pas l'école ou ne sont pas ma-riées (Graphique 4). À l'évidence, ces filles « ne sont pas oisives », mais il se pose une question troublante, relative à la manière dont elles font usage de leur temps. Puisque ces trois principales activités ne tiennent pas compte de nombre de filles appartenant à ce groupe d'âge, il est manifeste que les données disponibles sont terriblement incomplètes. Passentelles leur temps à des activités de loisir ou à des travaux domestiques ? Peut-être, aspect plus important, dans quelle mesure leur « oisiveté » est-elle imputable au manque d'opportunités?

\section{Les filles ne font-elles vraiment rien?} Le cas du Pakistan ${ }^{4}$

La situation des adolescentes au Pakistan constitue une anomalie pour la région et pour d'autres pays au même stade de développement et ce, pour trois raisons. Fait le plus important, l'âge au mariage (22 ans) est exceptionnellement tardif. Le mariage avant l'âge de 15 ans est rare et seules 23 pour cent des filles âgées de 15 à 19 ans sont mariées. En outre, seules 32 pour cent des filles âgées de 10 à 19 ans fréquentent
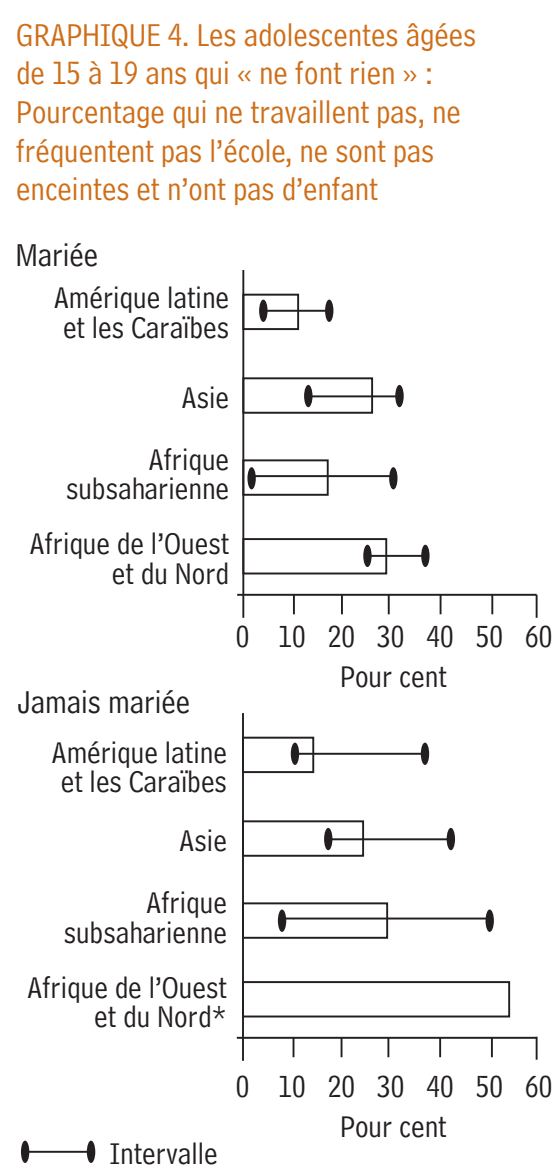

* La catégorie jamais mariée pour Afrique de l'Ouest et du Nord est seulement pour le Maroc.

Source : Calculs des données de l'EDS 1990-96. 
GRAPHIQUE 5. Statut scolaire, de travail* et de mariage des adolescents âgés de 10 à 19 ans, Pakistan

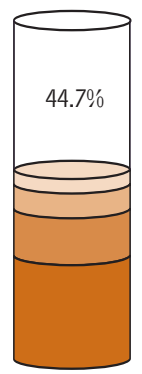

Filles

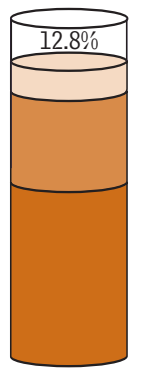

Garçons
Pas d'école, pas de travail, pas marié

7 Combinaison d'école, de travail et de mariage

\Seulement marié

$\square$ Seulement au travail

Seulement à l'école
*Pour qu'on les considère travailleurs, les filles et les garçons doivent travailler 10 heures ou plus par semaine dans un travail agriculturel ou non agriculturel, dans une ferme familiale ou dans un négoce familial.

Source : Calculs par Valerie Durrant basés sur des données du Pakistan Integrated Household Survey de 1991.

actuellement l'école. Enfin, le travail des filles (rémunéré et non rémunéré) ne représente pas non plus une grande proportion. Un pourcentage élevé de filles au Pakistan (45 pour cent) semblent « ne rien faire » de leur temps (Graphique 5).

Ce groupe est significatif, il représente un nombre important de filles qui n'ont pas de statut au niveau social. Elles sont étroitement surveillées et gardées à dessein à domicile. Elles sont seules, isolées, vulnérables et elles n'ont aucun lien avec les institutions sociales telles que l'école ou l'emploi. L'absence d'un groupe aussi important du tableau ainsi décrit, bat en brèche les croyances existantes relatives à la transition vers l'âge adulte.

\section{Compréhension des activités des filles}

La compréhension des activités des filles est au cœur des initiatives d'élaboration de politiques positives et efficaces et de programmation, destinées à améliorer leur si- tuation. Il est important de savoir comment et où les contacter afin d'initier des programmes intelligents. Le fait de savoir comment elles passent leur temps est crucial pour identifier les faiblesses inhérentes aux données actuelles qui occultent ou présentent de manière inexacte les activités économiques des filles.

À l'aide d'études quantitatives sur les données des enquêtes-ménages disponibles au niveau national, les chercheurs au Pakistan ont pu identifier les filles qui sont les plus susceptibles de ne rien faire. On trouve parmi elles : celles des zones rurales ; de la frontière nord-ouest et des provinces du Balochistan ; celles vivant dans les quintiles économiques inférieurs ; les filles dont les mères sont illettrées; et les filles âgées de 14 et 15 ans, l'âge auquel beaucoup d'entre elles ont abandonné l'école mais sont encore loin de l'âge du mariage, créant ainsi un décalage dans la transition vers l'âge adulte.

La recherche au Pakistan décrit la situation suivante de l'emploi du temps des filles : dans les zones rurales, le point culminant de « l'oisiveté » des filles apparaît durant la phase intermédiaire de l'adolescence. Les filles des zones urbaines qui ont

La compréhension des activités des filles est au cœur des initiatives d'élaboration de politiques positives et efficaces et de programmation, destinées à améliorer leur situation. 


\section{OUTILS}

A u Pakistan, la première tentative destinée à obtenir ces informations qualitatives s'est fondée sur les consultations des groupes de discussion thématique avec les adolescentes. Les chercheurs ont interrogé les adolescentes sur la formation scolaire, le travail, le mariage et la santé de la reproduction ; ce qu'elles souhaitent pour elles-mêmes ; et les opportunités dont elles disposaient en vue de satisfaire ces désirs ou aux obstacles auxquels elles sont en butte, à l'inverse. Il leur a également été demandé d'identifier au sein de leurs communautés, les changements potentiellement bénéfiques qui pourraient améliorer leurs possibilités d’atteindre leurs objectifs. Cette approche s'est avérée inefficace : les adolescentes ont donné des réponses socialement correctes que l'on trouve dans les «manuels scolaires » et ont parlé d'aspirations qui cadrent mal avec la réalité et elles n'ont presque rien révélé de la manière dont elles usaient de leur temps, des activités et des compétences auxquelles elles accordaient de la valeur, ni de la manière dont l'emploi actuel de leur temps s'inscrit dans la vie en général.

La deuxième tentative de recueillir des informations a davantage mis l'accent sur l'acquisition et le développement des compétences. Les adolescentes et leurs parents ont été interrogés sur les compétences qu’ils jugeaient importantes et utiles et sur la manière dont ils pouvaient les améliorer. Cela a permis de poser des questions sur la formation scolaire, le travail et le mariage et de susciter des réponses plus utiles. En réalité les filles aspirent fortement à fréquenter l'école et souhaitent avoir l'opportunité de s'engager dans des activités génératrices de revenus. Les chercheurs ont pu utiliser les incongruités contenues dans les réponses afin d'obtenir certaines informations. Les filles ont par exemple exprimé à plusieurs reprises leur souhait de terminer le cycle secondaire à l'âge de 15 ou 16 ans et de se marier à 22 ans. Il leur a été demandé ce qu'elles voudraient faire dans l'intervalle ; en réponse, elles ont mentionné les contraintes auxquelles elles font face, y compris une absence de structures, une mobilité restreinte et des opportunités limitées. À l'avenir, des profils détaillés de l'emploi du temps seraient très utiles. L'utilisation des activités initiées par les sujets interrogés - plutôt que la présentation d'un menu — et le fait d'interroger ces derniers davantage sur le « temps libre » et les activités de loisir, produiraient plus de données révélatrices ainsi que le ferait la prise en compte des variations saisonnières. Des questions isolées portant sur l'emploi ne donnent pas une image complète du tableau - les questions relatives à la formation scolaire, au mariage ou aux autres activités ne le font pas non plus. 
plus de possibilités de formation scolaire « ne font rien » à une phase plus tardive de leur adolescence. « L'oisiveté» n'est pas un produit ou une partie nécessaire de la transition, mais elle suit une évolution. Cependant, Iorsque les travaux ménagers sont inclus, le pourcentage de filles « qui ne font rien » baisse régulièrement avec l'âge aussi bien dans les zones rurales que dans les zones urbaines, ce qui indique que les filles font davantage usage de leur temps au fur et à mesure de l'adolescence. ${ }^{5}$

\section{"Oisiveté » ou travaux ménagers?}

Le rôle des travaux ménagers devient important lorsque l'on étudie le dilemme des filles qui «ne font rien ». Beaucoup de filles qui ne font rien en apparence, font en réalité beaucoup de travaux ménagers (presque exclusivement le linge, la cuisine et le ménage). Bien qu'il soit primordial de ne pas déprécier la valeur qui a finalement été accordée aux travaux ménagers, ils ne sont pas reconnus au plan social et n'apportent que peu d'avantages aux filles en matière de renforcement des compétences et de développement personnel. Les filles travaillent-elles comme domestiques parce qu'elles n'ont rien d'autre à faire ou ne sont-elles pas capables de tirer parti des autres opportunités en raison des travaux ménagers qu'elles doivent effectuer?

\section{Valeur du temps consacré aux loisirs}

L'autre question importante consiste à savoir si les loisirs constituent une activité appréciée ou non et s'ils passent inaperçus en vue de quantifier l'emploi du temps. Davantage d'attention doit également être

\section{En dernière analyse, il est difficile de trouver des filles qui « ne font rien ». En réalité, les plus isolées sont les plus occupées.}

accordée à la façon dont les filles perçoivent la qualité du temps qu'elles consacrent à différentes activités. Si les chercheurs et les tenants des politiques veulent améliorer la vie des adolescentes, à quelles contraintes sont-ils confrontés ?

Les filles ne sont pas " oisives"

En dernière analyse, il est difficile de trouver des filles qui « ne font rien ». En réalité, les plus isolées sont les plus occupées. Elles étaient trop occupées pour participer à l'entretien (au moins du point de vue de leurs parents) et il a donc été difficile de les localiser. Cet aperçu s'est révélé instructif en soi car il permettait d'avoir une idée de ce qu'elles faisaient, en l'occurrence des travaux ménagers. Une autre observation importante émanant des groupes de discussion était que les filles au Pakistan n'ont aucun concept du « temps libre ». Il semble qu'elles consacrent aux activités domestiques le temps dont elles ne font pas usage. Par rapport aux garçons surtout, les filles ne sont pas en réalité « oisives ».

\section{Nouvelles interrogations}

D'autres questions importantes demeurent; par exemple, on ne sait pas très bien depuis combien de temps les adolescentes qui « ne font rien » sont dans cette situation et l'on ne sait pas grand chose de la manière dont 
« l'oisiveté » durant l'adolescence a une incidence sur les perspectives d'avenir des filles. Les travaux ménagers des adolescentes viennent-ils en complément du travail des adultes? Les adolescentes mariées font-elles plus de travaux ménagers en termes relatifs et en termes absolus que celles qui ne sont pas mariées ? Il est peut être plus révélateur d'examiner les autres types de travaux ménagers effectués par les adolescentes mariées par rapport aux adolescentes non mariées. Font-elles la cuisine, le ménage, le linge et les autres activités dans les mêmes proportions ? Il est sensiblement nécessaire de mener une recherche qui place la participation des filles à diverses activités ou leur manque de participation, dans une perspective à long terme, notamment par rapport aux expériences acquises et à leurs futures opportunités.

\section{Travailler? Les adolescents et la main d'œuvre égyptiennee ${ }^{6}$}

En 1998, le Forum sur les recherches économiques a initié en collaboration avec le Population Council, une enquête sur le marché du travail représentative au niveau national, dans le cadre d'efforts destinés à déterminer où se situent les opportunités et d'où elles émergent sur le marché du travail égyptien.

Les jeunes — plus de 13 millions âgés de 15 à 24 ans — constituent actuellement la frange la plus importante de la population égyptienne. La majorité vit dans les zones rurales particulièrement en Basse Égypte. Quatre-vingt-dix pour cent d'entre eux ont fréquenté l'école brièvement (42 pour cent sont actuellement inscrits) bien que 16 pour cent soient illettrés, au nombre desquels on compte un cinquième étant de sexe féminin.

Trente deux pour cent de ces 13 millions de jeunes - presque 4,2 millions sont actuellement sur le marché du travail ; ils sont soit employés, soit au chômage et à la recherche d'emploi. (Dans l'enquête, une personne sans emploi est définie comme quelqu'un qui désire travailler, est capable de travailler et recherche activement un emploi - définition courante en Égypte.) Même si plus de deux fois plus de jeunes hommes que de jeunes filles (43 pour cent et 19 pour cent, respectivement) participent à la vie active, le fossé des sexes est moins grand parmi les jeunes que dans l'ensemble de la population active. Il existe cependant de graves disparités entre les sexes, qui font que le chômage est trois fois plus élevé chez les filles que chez les garçons. Dans l'ensemble, les taux de participation sont plus élevés dans les zones rurales que dans les zones urbaines, plus élevés dans les zones rurales de la Basse Égypte (à 37 pour cent) et plus faibles à Alexandrie et à Suez. Le chômage est plus élevé parmi les jeunes ayant un niveau d'éducation intermédiaire et parmi ceux des zones rurales.

\section{Tendances de l'emploi salarié}

En étudiant les jeunes âgés de 20 à 24 ans dans la vie active, on note que :

- 48 pour cent exercent des activités rémunératrices (dont 40 pour cent d'hommes et 8 pour cent de femmes); et 


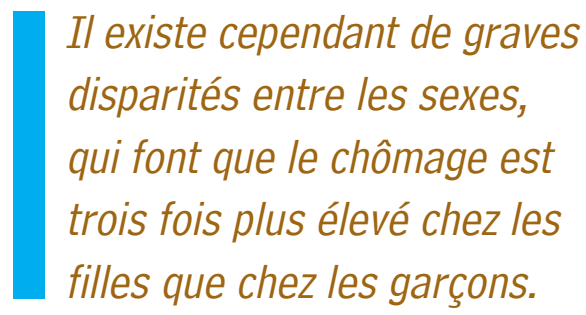

- Entre 1988 et 1998, l'emploi salarié a augmenté de 12 pour cent parmi les jeunes gens et baissé de 21 pour cent parmi les jeunes femmes, ce qui a accru le fossé des sexes de 28 pour cent.

Les résultats indiquent également qu'il y a eu durant cette période, un transfert impressionnant de l'emploi salarié des zones urbaines vers les zones rurales une augmentation de 24 pour cent parmi les jeunes femmes contre 11 pour cent parmi les jeunes hommes. Cependant, cet accroissement n'a pas compensé la baisse notée dans les zones urbaines, ce qui explique la baisse du taux global de participation chez les jeunes femmes.

\section{Où trouver des opportunités d'emploi ?}

Un examen de la répartition sectorielle de l'emploi rémunéré chez les jeunes révèle que la plupart d'entre eux sont employés dans les structures privées. L'enquête indiquait également leur lieu de travail dans le secteur privé. Les opportunités sont toujours disponibles dans le secteur manufacturier même si elles s'amenuisent lentement et celles de la main d'œuvre agricole ont également diminué. Les secteurs dynamiques comprennent la construction, le commerce, le transport, les finances et les services. Pour les hommes, les nouvelles professions sont en règle générale issues de la production, des services, de la vente et dans une moindre mesure de la technologie et de la science.

La situation est cependant quelque peu différente pour les femmes. De manière plus perceptible, les opportunités ont accru dans le secteur manufacturier et les disparités entre les niveaux d'emploi des secteurs privé et public sont nettement plus faibles que chez les hommes. ${ }^{7}$ Les opportunités dans les domaines du commerce et des finances augmentent aussi, bien qu'elles soient dans la majorité des cas limitées à celles qui ont bénéficié d'une éducation. Les opportunités dans les domaines scientifique et technique, qui incluent la profession infirmière et l'enseignement, augmentent aussi, de même que le travail de bureau, la vente et la production. Les opportunités dans le domaine des services se réduisent pour les jeunes femmes bien que cela puisse être dû aux changements apportés aux classifications des emplois dans les secteurs public/privé.

\section{Opportunités d'emploï : la quantité par rapport à la qualité}

Le problème de la quantité par rapport à la qualité est important lorsque l'on examine les secteurs en pleine croissance. Les missions de travail temporaire ont augmenté de plus de 100 pour cent, ce qui indique que les nouveaux emplois sont en règle générale de nature temporaire ; il existe moins d'opportunités pour les emplois contractuels. Les niveaux des avantages ont 
baissé alors que le nombre d'heures de travail hebdomadaire a accru. Il est manifeste qu'une grande partie des opportunités de nouveaux emplois repose sur la section informelle du secteur privé.

\section{Quel est l'âge des adolescents égyptiens qui travaillent?}

Les données de l'enquête nationale menée en 1997 sur les adolescents ont fait apparaître que 60 pour cent des adolescents ayant un emploi non rémunéré et 35 pour cent de ceux qui ont un emploi rémunéré ont moins de 15 ans (l'âge minimum légal pour travailler) (El-Tawila et al. 1999). Un nombre moins élevé de filles que de garçons ont un emploi rémunéré, même si les taux d'emploi rémunéré sont généralement constants entre 10 et 19 ans pour les deux groupes, avec un accroissement régulier et culminant des taux d'emploi pour les garçons à 11,14 et 18 ans. En particulier, il apparaît que ces points culminants correspondent aux changements survenant durant la scolarité : l'école primaire finit et l'école préparatoire commence vers 11 ans, l'école préparatoire finit et l'école secondaire débute vers 14 ans et l'école secondaire prend fin vers 18 ans.

\section{Âge d'entrée en activité}

Quarante pour cent des jeunes hommes qui sont présentement sur le marché du travail, ont commencé entre 11 et 16 ans alors que 42 pour cent des jeunes femmes ont commencé à travailler entre 16 et 20 ans. L'âge moyen de l'entrée en activité tourne autour de 15 ans pour les garçons et 17 ans pour les filles.

\section{Scolarisation et travail :}

une proposition dichotomique?

En Égypte, il y a à peine 20 ans, il était généralement admis que les enfants dont le travail était fructueux pour les parents étaient privés d'éducation. L'on pensait que ces enfants travaillaient comme ouvriers agricoles ou aux côtés de leurs parents comme apprentis alors que les autres allaient à l'école. Ceux issus de ménages pauvres et qui fréquentaient l'école abandonnaient l'école à un stade précoce.

\section{Il existe un lien évident entre les mauvais résultats et les abandons - les élèves ne quittent pas l'école pour chercher un emploi ou pour se marier. Ils décrochent parce qu'ils estiment qu'ils ont de mauvais résultats.}

Si l'on examine l'emploi à la lumière des inscriptions à l'école, une des tendances qui se dessinent de manière constante est que les garçons et les filles qui ne sont pas à l'école travaillent — avec ou sans rémunération - en plus grand nombre que leurs camarades qui vont à l'école. On note aussi qu'un nombre important de garçons et de filles déclarent être employés alors qu’ils fréquentent aussi l'école, ce qui indique que les deux activités ne sont pas essentiellement incompatibles. 


\section{Principale raison pour abandonner l'école}

Les données de l'enquête nationale ont révélé beaucoup d'informations sur les raisons pour lesquelles les étudiants quittent l'école. Plus d'un tiers des adolescents qui ont décroché ont cité les mauvais résultats scolaires comme étant la principale raison. C'était le cas des garçons et des filles de toutes les cinq régions d'Égypte et ce n'était pas lié au statut socio-économique d'une famille. Il existe un lien évident entre les mauvais résultats et les abandons les élèves ne quittent pas l'école pour chercher un emploi ou pour se marier. Ils décrochent parce qu'ils estiment qu'ils ont de mauvais résultats. Par conséquent il est important de comprendre les facteurs qui influencent les résultats scolaires d'un adolescent. Dans une analyse à plusieurs variables, après avoir vérifié les quatre facteurs les plus importants liés aux résultats scolaires (sexe, région de résidence, statut socio-économique et statut professionnel), il est apparu qu'il n'existe pas de différences entre les sexes ou de différences régionales significatives en matière de résultats scolaires. Au contraire, et curieusement, le facteur le plus déterminant est le statut socio-économique du ménage de l'élève. Par exemple, les enfants issus de familles pauvres sont 2,5 fois plus susceptibles de repasser un examen ou de redoubler une classe. Le deuxième facteur le plus important concernait le statut professionnel : les étudiants travaillant étaient 1,6 fois plus susceptibles de redoubler une classe ou de repasser un examen que ceux ne travaillant pas. Ces informations sont cruciales étant donné qu'un tiers des garçons et un dixième des filles qui fréquentent l'école travaillent aussi.

\section{LE TRAVAIL DES FILLES ET L'ENVIRONNEMENT POLITIQUE ET NORMATIF}

\section{Quelles sont les contradictions}

entre le travail et l'éducation ? ${ }^{8}$

Le modèle égyptien fait apparaître que du point de vue politique, il semble qu'il existe une contradiction entre l'emploi et la scolarisation, notamment durant la phase intermédiaire de l'adolescence : lorsque les besoins en matière d'enseignement obligatoire sont moins stricts les opportunités d'emploi sont en concurrence avec les études. L'atelier s'est efforcé de comprendre ces contradictions et les discussions se sont articulées autour des questions qui suivent: ${ }^{9}$

- De quelle manière l'éducation prépare-t-elle les jeunes à un emploi utile?

- Existe-t-il des compromis significatifs entre le travail et l'éducation ?

- Ce rapport de cause à effet est-il uniquement négatif ? Ou également positif? 
- Quels sont à court et à long termes, les compromis entre le travail et l'éducation?

- Comment résoudre au sein des communautés pauvres et des ménages démunis, cette contradiction entre la nécessité de répondre aux besoins à court terme et les avantages à long terme de l'éducation comme moyen de renforcer le capital humain?

- Existe-t-il à court terme des opportunités de meilleur emploi pour les adolescents ? Ces opportunités concurrencent-elles les avantages à plus long terme que l'éducation offre aux familles qui ont besoin de revenus?

Étant donné le nombre assez important de jeunes qui travaillent dans le secteur informel et les investissements que les pays ont placés en eux, nous devons demander si les jeunes peuvent y acquérir des qualifications compétitives. Sinon, où peuvent-ils acquérir les compétences nécessaires à un emploi bien rémunéré ? Lorsque les élèves abandonnent l'école, ils sont privés des opportunités qu'elle offre et n'améliorent pas nécessairement la possibilité de faire valoir leurs compétences sur le marché du travail grâce à une expérience professionnelle plus élargie. Les adolescents qui travaillent dans le secteur informel sont particulièrement susceptibles d'être non qualifiés. En Égypte comme dans d'autres pays, les adolescents qui allient scolarité et emploi non rémunéré travaillent en général comme ouvriers agricoles, ce qui les expose à des risques sanitaires

\section{RAISONS POUR LESQUELLES LES FILLES POURRAIENT NE PAS FRÉQUENTER L'ÉCOLE}

\section{La pauvreté}

- On a besoin des filles à la maison pour faire les travaux ménagers, travailler dans les champs ou aider dans l'entreprise familiale.

- Les frais d'éducation excèdent ce que les parents peuvent payer.

\section{La culture et la société}

- L'école est perçue comme une faible priorité pour les filles.

- Les filles se marient tôt.

- Les filles tombent enceintes.

- La mobilité des filles est limitée.

\section{La qualité de l'école}

- Les écoles sont peu nombreuses et leur effectif est pléthorique.

- Le préjugé sexiste se retrouve dans les programmes d'études, les méthodes et les ouvrages pédagogiques.

- Les enseignants et les parents ont une attitude discriminatoire.

- Les enseignants et les garçons harcèlent les filles.

- Il est difficile pour les filles de se rendre à l'école compte tenu de la distance entre la maison et l'école et l'accès insuffisant au transport. 
(par exemple, contact avec engrais chimiques et pesticides) sans nécessairement leur fournir des compétences particulières qui leur permettront de chercher de meilleures opportunités à l'avenir. Les adolescents qui allient scolarité et travail rémunéré ne sont pas souvent en mesure d'acquérir des qualifications par l'intermédiaire de l'apprentissage parce qu'ils ne sont pas disponibles pour travailler durant les longues heures normales de travail subalterne qui leur serait probablement assigné.

L'avenir des jeunes filles pourrait être mieux assuré grâce à des politiques et programmes qui instaurent un équilibre entre les besoins actuels en matière de subsistance et les futures opportunités ainsi que les avantages à long terme offerts par l'éducation. L'investissement dans l'éducation améliore les perspectives d'emploi pour les filles et les garçons, bien que dans nombre de cas, l'éducation des filles apporte de plus grands bénéfices que celle des garçons. L'éducation des filles offre un certain nombre d'avantages aux filles mêmes, à leur présente et future famille ainsi qu'à la communauté. Les filles ont particulièrement besoin d'être éduquées pour se préparer à participer pleinement et à égalité au développement politique, social et économique de leur société.

Cependant, 73 millions de filles en âge d'aller à l'école primaire n'ont toujours pas accès à l'éducation de base. Dans les pays les moins avancés, seuls 13 pour cent des filles et 22 pour cent des garçons sont inscrits à l'école secondaire. Où sont les filles lorsqu'elles ne sont pas à l'école ? Elles travaillent peut être à la maison ou dans les champs ; elles travaillent hors du domicile, au marché ; elles vivent dans les rues, en situation de détresse ; elles sont enceintes et renvoyées de l'école ou trop pauvres, trop affamées ou trop malades pour aller à l'école.

Comme il fallait s'y attendre, il existe une corrélation très positive entre la fréquentation scolaire et les revenus du ménage, particulièrement pour les filles. Dans la majorité des pays, les adolescentes ont tendance à se retrouver hors des systèmes scolaires formels et non formels en raison de la pauvreté et de la nécessité de travailler pour contribuer au revenu de leur famille. En règle générale, le faible accès à l'éducation est endémique dans les régions, communautés et ménages affectés par la pauvreté à travers le monde.

Et cependant, comme le montrent les données sur l'Égypte, alors que la décision même de travailler plutôt que de fréquenter l'école est due à la pauvreté, beaucoup d'adolescents allient les deux. Dans certaines parties du monde, notamment en Afrique subsaharienne, cette évo-

Comme le montrent les
données sur l'Égypte, même
si la corrélation négative
entre la fréquentation
scolaire et le travail est une
conséquence de la pauvreté,
beaucoup d'adolescents
allient les deux.


lution est quelque peu une norme sociale et les écoles prennent des dispositions à cet effet. Les adolescents qui travaillent ont souvent de meilleurs résultats scolaires et leur travail leur permet de prendre en charge leur éducation et dans bien des cas celle de leurs frères et sœurs. Les adolescents peuvent apprendre et ils apprennent effectivement de leur travail grâce à l'apprentissage et à des programmes de formation professionnelle soigneusement conçus.

\section{Réductions des corrélations négatives}

\section{entre le travail et l'école?}

Il existe un grand nombre d'opportunités de programmes qui prennent en charge les divers besoins des adolescents en matière de scolarité et d'emploi. Elles comprennent notamment :

- Des dispositions assurant un horaire flexible à l'école et au travail ;

- Un soutien et des incitations économiques aux parents qui permettent à leurs enfants de fréquenter l'école au lieu de travailler ;

- Des écoles «parallèles » qui proposent une éducation non formelle ce qui peut réduire le manque à gagner tout en augmentant l'accessibilité ;

- La programmation d'enseignements « hors de l'université » au profit des jeunes marginalisés et défavorisés ;

- Une éducation adaptée aux besoins des adolescents en matière d'emploi ; et

- Des initiatives visant à éliminer les abus et l'exploitation au travail grâce à la mise en place de milieux de travail sûrs et la création d'opportunités.
Promotion de milieux de travail sûrs et productifs pour les adolescentes: normes de I'UNICEF et du BIT et

\section{l'industrie de la confection au}

\section{Bangladesh ${ }^{10}$}

L'industrie de la confection s'est rapidement développée au Bangladesh au cours des années 90, passant de 50 usines employant 10000 ouvriers en 1983 à 2460 usines employant 1,4 million d'ouvriers en 1998, dont 85 pour cent sont de sexe féminin. La croissance de l'industrie a été accompagnée d'un débat non moins croissant sur ses « retombées ». Pendant que nombre de gens la considéraient comme un bel exemple d'initiative de croissance économique du secteur privé basée sur le commerce, l'Occident l'examinait sous toutes les coutures compte tenu du sentiment que les ouvriers étaient insuffisamment rémunérés et s'éreintaient dans des conditions inhumaines. En outre, les Américains - qui assurent 85 pour cent des exportations de vêtements du Bangladesh - ont été induits à penser qu'un « ouvrier d'usine de confection » est en langage courant « un enfant qui travaille ». Des acheteurs animés de bonnes intentions, ont imaginé des enfants de 10 ans travaillant 18 heures par jour dans des usines plongées dans l'obscurité et gagnant des centimes par heure, se sont en définitive joints au Congrès américain et aux associations de consommateurs pour boycotter les articles qu'ils considéraient avoir été fabriqués dans de telles conditions.

En réalité, compte tenu des données de 1993, 6,3 millions d'enfants travaillaient 
au Bangladesh, dont 96 pour cent dans le secteur informel et seulement 4 pour cent dans le secteur formel. Les meilleures estimations - bien que toutes les données statistiques sur le travail, notamment celles sur le travail des enfants, doivent être considérées comme suspectes - indiquaient que seuls 50000 à 70000 enfants étaient employés dans les usines de confection, soit environ 5 à 7 pour cent de la main d'œuvre des usines de confection. Cependant, parce qu'ils craignaient des mesures dissuasives supplémentaires de la part des États-Unis, les directeurs d'usine ont affirmé qu'à l'horizon 1994, le secteur du vêtement n'emploierait plus aucun enfant. Du jour au lendemain, la panique a conduit par mesure de sécurité, au licenciement de plus de 50000 travailleurs mineurs. Ces enfants n'ont eu d'autre choix que de chercher un autre emploi, qui serait moins bien rémunéré et dans certains cas moins sûr. Et quand même, environ 20000 enfants demeuraient des employés de l'industrie après le dernier délai. Certaines dispositions devaient être prises à leur égard. En conséquence, le secteur privé, I'UNICEF et ensuite le BIT ont élaboré un Protocole d'entente $(\mathrm{PE})$ y afférent.

\section{Protocole d'entente}

\section{UNICEF/BIT/BGMEA}

Au début de 1994 et jusqu’à la fin de 1995, I'UNICEF et le BIT ainsi que l'Association bangladaise de confection et d'exportateurs de vêtements (BGMEA) ont commencé à faciliter la conclusion d'un accord tripartite - le premier du genre à intéres- ser le secteur privé - en vue créer un programme de grande envergure, destiné aux employés mineurs de l'industrie de la confection. Nombre de stratégies différentes ont été prises en considération. Le modèle sur lequel I'UNICEF et le BIT avaient porté leur choix proposait que les enfants fussent autorisés à demeurer employés dans les usines, en travaillant des journées de 6 heures et en fréquentant l'école pendant deux ou trois heures dans des établissements dirigés par des organisations gouvernementales locales. L'ambassade des États-Unis a manqué d'entériner cette formule, exigeant plutôt que les enfants soient purement et simplement écartés des usines. En définitive, les dispositions suivantes figuraient dans le Protocole d'entente signé le 04 juillet 1995 :

- Le BIT mènerait une prompte enquête d'évaluation dans l'ensemble des usines en vue de déterminer l'ampleur du travail des enfants.

- À la suite de l'évaluation, les enfants âgés de moins de 14 ans seraient retirés des usines et placés dans un programme d'éducation mis en œuvre par l'UNICEF.

- Le BGMEA a accepté de ne pas licencier les travailleurs de moins de 14 ans avant la fin de l'évaluation et la création d'écoles appropriées.

- À l'avenir, les travailleurs recrutés seraient âgés d'au moins 14 ans.

- Les enfants qui étaient précédemment employés dans les usines et fréquentaient désormais les écoles, recevraient une allocation de $300 \mathrm{Tk}$ 


\section{LE RÔLE DU BIT}

' adoption et la supervision des conventions et recommandations régissant le travail Linternational qui représentent un consensus international sur les normes minimales de travail, constitue l'un des outils majeurs à la disposition du BIT, pour l'amélioration de la législation et des pratiques de ses états membres. La Convention 182 est la première norme internationale régissant le travail des enfants, qui stipule expressément qu'une attention particulière doit être accordée aux filles. Dans la Recommandation (No 146) annexée à la Convention, le BIT attire l'attention sur les situations relatives au travail non réglementé ou clandestin — tel que les emplois du secteur informel où les filles sont exposées à des risques particuliers. Les gouvernements qui ont ratifié la Convention sont censés mettre en place des comités nationaux de surveillance du travail des enfants dans tous les secteurs à l'échelle locale et s'assurer que les interventions atteignent le groupe cible.

La Convention de 1973 sur l'âge minimum (Convention 138) établit au moins trois âges minimums pour l'entrée dans la vie active. En premier lieu, l'Article 2 stipule que l'âge minimum pour prétendre à un emploi rémunéré doit coïncider avec l'âge où on termine la scolarité obligatoire (qui varie en fonction des pays, mais se situe généralement autour de 15 ans). L'Article 3 stipule qu'une personne devrait être âgée d'au moins 18 ans avant de se vouer à un emploi susceptible de mettre en danger la santé, la sécurité, et la moralité des jeunes, comme le travail en souterrain ou dans les espaces clos. Des exceptions destinées à permettre aux jeunes de 16 ans de se consacrer à de tels emplois peuvent être accordées après un processus de consultation entre les partenaires sociaux (gouvernement, syndicats et patronat). L'Article 7 traite du travail non pénible. Compte tenu de l'âge fixé à l'Article 2 pour un emploi rémunéré, il est possible de commencer un travail non pénible deux ans plus tôt, par exemple, à 13 ans dans les pays où la scolarité obligatoire prend fin à 15 ans.

La Convention sur les pires formes de travail des enfants (Convention 182) a été adoptée en 1999. Les états qui l'ont ratifiée s'engagent à bannir et à éliminer les pires formes de travail des enfants pour ceux qui sont âgés de moins de 18 ans, en utilisant la définition que la Convention des Nations Unies relative aux droits de l'enfant donne de «l'enfant ». Les pires formes de travail des enfants se répartissent en quatre catégories. On compte parmi ces catégories, (1) le travail servile, y compris le travail forcé, la servitude pour dette et la participation forcée à un conflit armé ; (2) la prostitution et la pornographie ; (3) la participation à des activités illicites, avec un accent particulier sur la production et le trafic de drogue et (4) le travail qui pourrait avoir des effets néfastes sur la santé, la sécurité et la moralité des enfants.

Source : Résumé présenté par Theresa Smout. 
(environ 6 USD) par mois (une portion de ce qu'ils avaient gagné), dont 50 pour cent ont été subventionnés par le BGMEA. Le BIT a également participé aux allocations grâce à son Programme international pour l'élimination du travail des enfants.

Le programme a démarré en 1996 et a connu beaucoup de difficultés. Les esprits critiques se sont demandé pourquoi le BIT s'était engagé dans un programme qui ne rejetait pas d'emblée le travail des enfants. En théorie, il était souvent difficile de déterminer l'âge de nombre de jeunes travailleurs dans la mesure où le Bangladesh ne dispose pas de système formel d'enregistrement des naissances. Au départ, certains propriétaires d'usines ont refusé de coopérer dans le cadre de l'évaluation. En outre, l'allocation mensuelle était insuffisante, ce qui a amené certains participants à chercher des emplois à temps partiel. Au bout du compte, les structures destinées à l'amélioration des compétences et à la formation demeurent peu satisfaisantes.

\section{Perspectives}

L'initiative arrivera à son terme en 2000. Le programme a déjà accordé des diplômes à plusieurs enfants. Certains veulent retourner dans l'industrie de la confection, certains veulent poursuivre leurs études et d'autres veulent aller dans d'autres secteurs industriels. En dépit des difficultés rencontrées, ce projet a prouvé qu’il était possible de combiner salaire et études.

\section{COMMENT LES JEUNES ADOLESCENTES PERÇOIVENT-ELLES LEURS CONDITIONS DE TRAVAIL ?}

\section{COMMENT LE TRAVAIL CONTRIBUE-T-IL À LEUR «ADOLESCENCE»?}

POINT DE VUE DE TROIS PAYS

\section{Égypte ${ }^{11}$}

Le Population Council a mené des études de cas (les travaux étaient en cours au moment où s'est tenu l'atelier) sur les jeunes femmes actives des milieux urbain et rural d’Égypte afin d'avoir une meilleure idée de leurs raisons de travailler, le type d'emploi qu'elles occupent et la manière dont elles perçoivent leur emploi. Dans les études de cas, les jeunes femmes sont représentatives des jeunes femmes actives des gouvernorats où le Council a mené ces 
recherches - elles travaillent principalement dans des usines de confection et de petits ateliers qui fournissent la plupart des opportunités d'emploi du secteur formel dans plusieurs régions.

\section{Pourquoi les jeunes femmes travaillent-elles?}

Bien que les recherches du Council fussent toujours en cours, certaines tendances étaient déjà apparues. D’abord, il était clair que la pauvreté était la motivation essentielle — bien qu'elle ne fût pas la seule — des jeunes femmes à travailler. Les jeunes femmes apprécient aussi la mobilité accrue dont elles jouissent en tant que travailleuses. Elles déclarent que le travail leur donne la possibilité des se faire des amis et des camarades, éléments sociaux qui autrement sont difficiles à intégrer dans leur vie quotidienne une fois l'école terminée en raison de leur mobilité limitée. Les jeunes femmes reconnaissent aussi que le travail est une manière productive de passer leur temps et qu'à la condition de « travailleuse » s'attache de la valeur (et de l'argent), tout en faisant noter que le fait de travailler leur évite de rester à la maison toute la journée.

\section{Quel type de formation les filles} reçoivent-elles et où ?

La majeure partie des filles sondées dans les études de cas exercent un emploi non spécialisé dans les magasins et usines de confection. Certaines jeunes femmes qui travaillent dans les zones franches du secteur formel ont la possibilité de béné- ficier d'une formation hors du lieu de travail selon le principe de la rémunération des services. Peu de filles bénéficient d'une formation dans les usines parce que très souvent, on leur demande de signer un contrat dans lequel elles promettent de travailler à l'usine pendant six ans au minimum, sans considération de leur salaire ou traitement - une sorte de travail servile lié par contrat à long terme.

\section{Conditions de travail}

Les conditions de travail des jeunes femmes sont souvent difficiles. Celles qui travaillent dans les usines de confection déclarent travailler de longues heures par rapport aux salaires qu'elles reçoivent. Cependant, elles sont en majorité satisfaites de leurs salaires même s'ils sont faibles. Ainsi que l'a dit une femme, « un faible salaire vaut mieux que pas de salaire du tout ». Plus exactement, les jeunes femmes sont contrariées par l'épuisement physique quelles ressentent lorsqu'elles travaillent dans les usines. En général, les travailleuses ont droit à une heure de repos durant la journée et elles ne sont autorisées à s'asseoir que pendant leurs pauses. Elles doivent souvent faire des heures supplémentaires sans compensation. De plus, les congés de maladie ne sont pas autorisés et la sécurité sociale ainsi que l'assurance maladie ne sont pas toujours garantis. Les conditions de travail désagréables, au-dessous de la norme, semblent renforcer la perception des jeunes femmes selon laquelle leur expérience du travail formel sera une entreprise 
de courte durée. En outre, peut-être parce que la plupart d'entre elles n'ont pas de projets à long terme dans le marché du travail, elles se perçoivent comme étant " bonnes à jeter ». Elles ont le sentiment d'avoir été recrutées sans difficulté et qu'elles peuvent par conséquent être renvoyées aussi facilement.

\section{Limite des opportunités offertes aux tra-} vailleuses : document de non-objection À Port-Saïd, sur l'ordre de la direction de l'usine, la Businessmen's Society a créé un mécanisme aux termes duquel les travailleuses doivent en réalité être autorisées à quitter leur emploi dans une usine pour signer un contrat dans une autre. Alors que les femmes sondées dans les études de cas affirment que leurs camarades et elles sont satisfaites de leurs conditions de travail, il arrive parfois qu'une employée veuille changer de travail. Pour ce faire, elle doit présenter au nouvel employeur ou à l'employeur potentiel, un document de non-objection, indiquant qu’elle a été " libérée » d'une autre usine qui « ne s'oppose pas » à ce qu'elle cherche un emploi ailleurs. Sans un document, la seule possibilité est de ne pas travailler pendant six mois, une éventualité à laquelle la majorité des jeunes femmes ne peuvent se permettre de faire face. Le plus souvent, les employeurs refusent de donner suite à la demande d'un travailleur qui sollicite le document.

\section{Droits des travailleurs}

La plupart des jeunes femmes sondées dans les études de cas ne connaissent pas leurs droits en tant que travailleuses. Celles qui les connaissent ont conscience de leurs limites et ne sont pas disposés à exiger leur garantie. L'une des conséquences de cette situation est que la majorité des travailleurs considérés dans l'étude de cas, n'ont pas d'assurance maladie même si elle est supposée être garantie avec l'emploi. Le harcèlement sexuel constitue une autre violation des droits et bien qu'il ne soit pas rare au travail (particulièrement dans les petites structures hors usine), il se manifeste plus souvent dans les rues, au moment où les femmes se rendent au travail ou en reviennent. II n'existe pas de syndicat et pas de négociation collective et ni le gouvernement, ni les ONG ne jouent un rôle de réglementation.

Les liens de parenté ou les relations pseudo-familiales compliquent souvent les relations entre employeurs et employés. Les supérieurs hiérarchiques de sexe masculin agissent parfois comme un père ou un grand frère devant les jeunes employées qui à leur tour, agissent avec déférence et timidité. Plus la hiérarchie de travail est paternaliste, moins la travailleuse remettra en cause le patron.

\section{Les travailleuses évaluent leur expérience}

En dépit des conditions de travail, parfois moins qu'idéales, les jeunes femmes déclarent indistinctement qu'elles ressentent une fierté et une estime de soi considérables grâce à leur travail. Elles aiment pouvoir faire des courses comme elles le désirent, puisqu'elles n'ont plus à deman- 
Le fait de donner de l'argent aux parents/frères/sœurs pourrait légitimer pour les jeunes femmes mariées un désir caché de rester dans la vie active pour d'autres raisons.

der la permission d'aller faire des achats. Lorsqu'on leur demande de comparer leur personnalité à celle de leurs camarades qui ne travaillent pas, presque toutes les jeunes femmes se perçoivent comme étant plus confiantes, plus autonomes et susceptibles d'avoir plus de choix au moment de trouver un partenaire conjugal. Beaucoup de femmes rapportent qu'elles économisent leurs gains afin d'acheter leur trousseau de mariage.

Traditionnellement plus une fille restait à la maison et menait une vie discrète, plus elle était honorée par sa communauté et plus elle aurait de la valeur en tant qu'épouse. Le coût croissant du mariage a obligé les jeunes filles à trouver un emploi afin de gagner elles-mêmes l'argent de la dot. Alors que le fait de voir une fille hors de la maison avait traditionnellement un effet négatif sur ses perspectives de mariage, sa capacité à gagner de l'argent lui a conféré de la valeur aux yeux de sa famille. Sa capacité à gagner de l'argent réduit les contraintes financières qu'un mariage impose à ses parents (particulièrement à la mère qui habituellement achète le trousseau de sa fille). Avec chaque livre qu'elle apporte à la maison, le
« fardeau » que constituait par le passé une fille à marier se transforme en « atout ».

Toutefois, les études de cas n'établissent pas clairement si le fait de travailler repousse l'âge auquel les filles se marient. En réalité, le travail peut faciliter le processus du mariage, s'il permet aux jeunes filles d'acquérir plus rapidement les biens dont elles ont besoin pour se marier. Étant donné que l'âge au mariage ne cesse d'augmenter indistinctement, les filles qui travaillent pourraient avoir plus de chance de trouver un mari dans un marché du mariage obstrué.

Les recherches ont en outre révélé que les jeunes filles mariées ayant grandi dans une pauvreté extrême et continuant à travailler après le mariage, le font généralement pour apporter leur appui à la maison familial ; dans certains cas, elles sont les seules sources de soutien financier. D'autre part, il est encore culturellement inacceptable qu'elles veuillent travailler parce qu'elles aiment cela, particulièrement une fois qu'elles sont mariées. Par conséquent, le fait de donner de l'argent aux parents/frères/sœurs pourrait légitimer un désir caché de rester dans la vie active pour d'autres raisons.

\section{La Jordanie ${ }^{12}$}

En Jordanie, la participation des femmes au marché du travail est faible et sa croissance a été lente par rapport à celle dans d'autres parties du monde en développement. Fait digne de remarque, tout porte à croire que la participation des jeunes femmes au marché du travail est de façon 


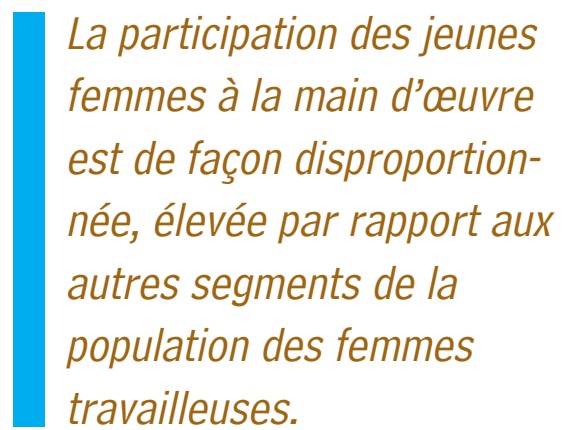

disproportionnée, élevée par rapport aux autres segments de la population des femmes actives. Les données de 1991 indiquent que 65 pour cent des femmes actives ont moins de 30 ans et que 60 pour cent sont célibataires. Le taux de croissance de l'emploi des femmes dépasse présentement celui des hommes. En même temps, l'âge au mariage a reculé pour les femmes et est passé de 17 ans en 1971 à 24 ans en 1995. L'accroissement des niveaux d'instruction et la diversification des opportunités d'emploi diversifiées pourraient être à l'origine d'une fraction de ce phénomène. Traditionnellement, les rôles et identités des femmes se sont répartis de manière précise dans une ou plusieurs catégories traditionnelles épouse, fille, sœur, mère. La visibilité croissante des femmes actives a cependant contribué à créer une nouvelle identité pour les femmes jordaniennes : celle d'une adulte célibataire, employée.

Afin de bien comprendre ce phénomène émergent, le BIT à Beyrouth a initié une enquête sur les employeurs de 36 institutions du secteur privé et une enquête par questionnaire portant sur 302 ménages dans 14 quartiers d'Amman. Un tirage au sort a identifié ces derniers à partir des ménages qui avaient participé à l'Enquête de 1991 sur l'emploi, le chômage, et la pauvreté de la Jordanie. Les participantes à l'enquête étaient âgées de 20 à 30 ans, célibataires, ne fréquentaient pas l'école et comprenaient aussi bien des femmes actives que des femmes au chômage. Les participantes avaient différents niveaux de revenus bien que la majorité fût composée de membres des professions libérales (des enseignantes souvent) travaillant dans le secteur privé.

L'éducation des filles est prestigieuse, l'emploi ne l'est pas

Dans toutes les catégories de revenu, la fréquentation d'un établissement d'enseignement supérieur par les filles est devenue liée au prestige. Et même dans ce cas, le choix d'éducation complémentaire que les filles font après l'école secondaire, continue d'être influencé par leur famille. Les femmes qui décident de poursuivre leurs études sont encouragées à poursuivre des études conformes aux rôles traditionnels assignés à chacun des sexes (comme l'enseignement.) Les femmes qui tentent de repousser ces limites rencontrent la résistance de leurs familles.

Alors que l'éducation des filles est raison de prestige, l'emploi ne l'est pas. L'emploi est perçu comme une menace potentielle aux normes traditionnelles, qui encouragent la ségrégation des sexes et le contrôle de l'autonomie des filles. Les jeunes femmes font souvent face à la résistance familiale lorsqu'elles veulent tra- 
vailler. Les familles insistent parfois pour que leurs filles travaillent à proximité du domicile ; qu'elles rentrent avant la tombée de la nuit ou qu'elles travaillent dans un environnement où il $n$ y' aurait que des personnes de leur sexe. Certaines industries tiennent compte de ces contraintes familiales et culturelles. Par exemple, les employeurs de grandes fabriques s'assurent que les journées de travail des femmes se terminent avant le coucher du soleil. D'autres fournissent un moyen de transport privé aux jeunes employées, afin de leur éviter les transports publics. Il n'est pas rare que les parents (le père en général) veuillent rencontrer un employeur potentiel afin de s'assurer de son « honneur » avant que la jeune femme ne s'engage pour un emploi.

L'importance accordée à un environnement de travail approprié signifie que les femmes sont plus susceptibles de travailler dans des lieux qu'ellesmêmes et/ou leurs familles - et par extension la collectivité - trouvent appropriés. En conséquence les salaires des femmes sont plus bas qu'ils ne devraient l'être et elles ne sont pas en position de négocier des salaires plus élevés.
Cependant, plusieurs familles trouvent encore les conditions de travail inacceptables. Même si les filles célibataires rentrent à la maison avant la tombée de la nuit, la journée de travail peut maintenir leurs filles éloignées de la maison pendant trop longtemps. Certains considèrent avec réticence une pause déjeuner de deux heures pour les travailleuses. Les lieux de travail mixtes ne sont pas tout à fait acceptés par certaines familles. Les employeurs ressentent le besoin de prouver que leurs entreprises sont exemptes de tout harcèlement et ils engagent parfois des femmes pour superviser les autres femmes. (En réalité le harcèlement sexuel n'est pas du tout aussi répandu que l'obsession à le prévenir.) Certaines femmes ont affirmé qu'elles avaient postulé pour des emplois où elles travaillaient avec des chefs ou des collègues à qui elles ne faisaient pas confiance et par conséquent, lorsqu'une offre d'emploi était prolongée, elles la refusaient. D'autre part, si une femme à la recherche d'un emploi a manifestement besoin de travailler, il est possible que l'employeur veuille profiter de la situation, la rendant vulnérable sur le lieu de travail. L'importance accordée à un environnement de travail approprié signifie que les femmes sont plus susceptibles de travailler dans des lieux qu'elles-mêmes et/ou leurs familles - et par extension la collectivité - trouvent appropriés. En conséquence les salaires des femmes sont plus bas qu'ils ne devraient l'être et elles ne sont pas en position de négocier des salaires plus élevés. 
Attitude, perceptions et satisfaction des travailleuses

L'enquête a tenté de déterminer dans quelle mesure l'expérience professionnelle pourrait ou non favoriser des changements au niveau personnel ou changer la perception que les femmes avaient des divisions selon le sexe et du mariage. Dans l'enquête, un nombre significatif de femmes pensaient qu'elles devraient occuper des emplois conformes à leur " nature féminine », comme l'enseignement, la couture ou l'artisanat. Quel que soit leur statut professionnel actuel, les jeunes femmes sondées ont indiqué qu'il était acceptable de travailler après le mariage - mais pas après avoir eu des enfants. Beaucoup d'entre elles ont toutefois reconnu l'importance de l'autonomie financière d'une épouse.

Bien que plusieurs femmes aient affirmé être peu satisfaites de leur emploi, elles apprécient la possibilité qu'il leur offre de quitter le domicile, de se faire des ami(e)s, de jouir d'une certaine liberté économique et de casser la monotonie de leur vie. Néanmoins, les jeunes femmes ont également rapporté avoir le sentiment qu'elles sont limitées par les options qui leur sont offertes plutôt que par l'autoperception de ce qu'elles savent être capables de réaliser. Les femmes qui ont des ambitions pour leur avenir sont jugées par les employeurs et par la société avoir des traits « masculins » qui peuvent réduire leurs chances de mariage. Ainsi, la hiérarchie des sexes en apparence impénétrable freine les ambitions de femmes. En somme, les résultats de l'enquête montrent que les graines de
« l'auto-différenciation » ont été semées, au moins parmi les femmes qui ont pris part à ces recherches. Parmi les participantes, des opportunités d'emploi plus nombreuses ont aidé les femmes à trouver des postes à partir desquels elles peuvent négocier un comportement normatif tenant compte du rôle des hommes et des femmes et leurs attentes.

\section{Le Bangladesh}

Ainsi qu'on l'a noté précédemment, le secteur de la confection au Bangladesh s'est fortement développé ces dernières années. Plusieurs politiques novatrices, au nombre desquelles la collaboration interinstitutions exceptionnelle susmentionnée entre l'UNICEF, le BIT et le BGMEA, ont contribué à son développement positif, en offrant des possibilités de scolarisation à la main d'œuvre enfantine qui avait quitté les usines de confection. Il y a de plus en plus d'indications selon lesquelles ce nouveau lieu de travail — qui attire un grand nombre de jeunes femmes célibataires dans un environnement dominé par la main d'œuvre masculine - a contribué à créer un nouveau stade, une adolescence qui par le passé n'existait pas et qui permet aux jeunes femmes de retarder le mariage. ${ }^{13}$

\section{Mutation des normes de travail et des} normes fondées sur l'égalité des sexes Selon la tradition bengalie, les filles sont considérées en âge d'être mariées dès qu'elles ont atteint la puberté. Plus de 75 pour cent des filles au Bangladesh sont mariées avant 18 ans. Ce mode de mariage est la raison sous-jacente du statut peu 
élevé et précaire dont les femmes souffrent toute leur vie. En outre, il y a souvent une différence d'âge importante entre les époux. Les filles ont très peu de choix quant aux rôles qu'elles peuvent jouer, en dehors de ceux d'épouses et de mères. Le coût des études est trop élevé et hors de portée des familles pauvres et sans doute l'autre aspect le plus important est la croyance très forte selon laquelle le mariage est sacro-saint et ne peut pas faire l'objet d'interventions.

Le mariage précoce a des conséquences significatives sur les filles et sur les tendances démographiques nationales. En fonction de l'exercice de répartition que John Bongaarts a effectué pour le Bangladesh, 80 pour cent de la future croissance démographique sera du à l'essor démographique. Cependant un recul de cinq ans de l'âge moyen au premier accouchement, contribuerait à réduire de 40 pour cent la croissance due à cet essor. Par conséquent, le recul de l'âge du mariage peut avoir des implications considérables sur les mutations démographiques.

Récemment les demandes de dot en espèces ont augmenté. Historiquement les musulmans, qui représentent 92 pour cent de la population, n'avaient pas, au nombre de leurs rites de mariage, le paiement d'une dot ; ce phénomène est plutôt apparu avec la rareté des possibilités de mariage et a désormais amené les familles à payer les futurs époux pour que leurs filles se marient. Au même moment, l'éducation gratuite pour les filles a augmenté leur fréquentation scolaire et la mécanisation agricole a réduit le

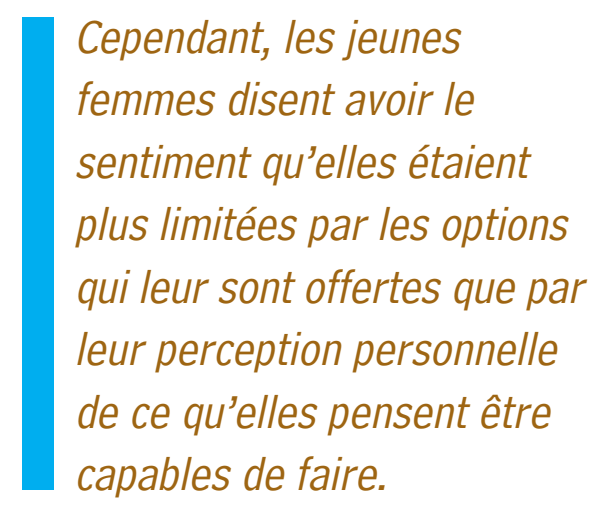

temps que les femmes et les filles consacrent à la production et à la transformation des aliments. Ces tendances distinctes ont coïncidé avec un accroissement des opportunités d'emploi dans le secteur formel, notamment dans celui de la confection, que les jeunes femmes célibataires occupaient le plus souvent selon les faits observés.

\section{Qui travaille dans le secteur de la confection?}

Le secteur de la confection est d'abord apparu vers la fin des années 70, s'est régulièrement développé durant les années 80 et a connu une croissance exponentielle dans les années 90. Composé principalement de grands ateliers de couture, le secteur de la confection est la plus importante source de devises pour le Bangladesh et aussi le secteur qui connaît la croissance la plus rapide dans une économie qui est par ailleurs, frappée de stagnation. En 1997, il comptait 1200000 employés (comparés aux 250000 de 1990). Un examen plus attentif du profil des travailleuses du secteur montre que :

- 78 pour cent ont moins de 25 ans ;

- 87 pour cent sont des migrantes en provenance des zones rurales du pays ; 


\title{
OPPORTUNITÉS D'ÉPARGNE POUR LES TRAVAILLEUSES DE L'INDUSTRIE DE LA CONFECTION
}

\author{
ien que les travailleuses de l'industrie de la confection aient une forte propen- \\ sion à épargner leurs gains, elles manquent généralement de moyens pour le
} faire. Considérant le nombre de travailleuses, elles représentent un marché important pour les banques. Parmi les moyens que les banques pourraient utiliser pour mettre leurs services à la disposition des travailleuses de l'industrie de la confection, on compte :

- La mise à disposition de guichets ou de filiales de banques qui s'occupent exclusivement des femmes ;

- L'ouverture de filiales de banques dans les locaux de l'usine ;

- La réduction des formalités d'ouverture de comptes d'épargne ;

- La mise en place d'heures d'ouverture spéciales pour les travailleuses d'usine afin qu'elles n'aient pas besoin de s'absenter du travail pour aller à la banque ; et

- L'adoption d'un modèle d'ONG consistant à envoyer un cadre de banque rendre visite aux femmes à domicile en vue de percevoir leur épargne.

Source : Recommandations présentées par Joachim Victor Gomes.

- 86 pour cent vivent avec les membres de leur famille (soit d'origine soit celle du mari) ; et

- 70 pour cent n'étaient pas mariées Iorsqu'elles ont commencé à travailler. Bien que leurs revenus soient très faibles, le profil de leurs dépenses montre que leur propension à épargner est remarquablement forte. Il est intéressant de noter que des familles entières et non seulement celles qui travaillent, déménagent pour habiter près d'une usine, afin que leurs filles puissent être embauchées.

Il semble que le travail retarde le mariage non seulement chez les filles qui travaillent mais aussi au sein des communautés qui envoient leurs filles travailler.
Dans la tranche d'âge des 20 à 24 ans, le pourcentage de filles qui se sont mariées avant l'âge de 20 ans est de 67 pour cent pour les travailleuses par rapport à 83 pour cent pour celles qui ne travaillent pas dans une région du Bangladesh qui envoie les filles en milieu urbain pour qu'elles travaillent dans les usines et à 92 pour cent pour les filles sans emploi dans des régions qui n'envoient pas les filles travailler. La tendance au retard du mariage des filles qui travaillent, peut en réalité influer sur les normes régissant le mariage au sein de la communauté pour les autres filles.

\section{Perception que les filles ont du travail}

Les filles indiquent que les opportunités de travail leur ont donné une nouvelle concep- 
tion de leur vie. Une travailleuse a expliqué qu'elle se voyait travailler et épargner pendant sept ans et qu'alors elle serait en mesure de s'acheter une maison et de payer sa dot. Pour elle, les années de travail sont une phase de transition - ses objectifs à long terme sont peut-être tout à fait traditionnels, mais elle les a présentés comme elle l'entend. Une autre travailleuse a décrit la manière dont elle peut mieux suivre la mode vestimentaire (comparées à ses amies mariées) et jouir d'une plus grande mobilité, en se déplaçant du village vers la ville et faisant remarquer qu'elle a plus confiance en elle-même comparée à ses amies qui sont sans emploi.

\section{FONDEMENTS DES CHOIX POLITIQUES ET PROGRAMMES DESTINÉS À ASSURER DES MOYENS DE SUBSISTANCE SÛRS ET APPROPRIÉS AUX ADOLESCENTES PLUS ÂGÉES}

\section{Comment trouver le cadre théorique de la politique adéquate ?14}

Considérant la réalité de la vie de tant de jeunes gens, y compris le nombre important de jeunes femmes qui à travers le monde, font partie de la population active à un titre quelconque, il est peut-être surprenant de voir que dans l'ensemble, les jeunes n'ont pas de cadre institutionnel dans lequel ils peuvent établir des moyens de subsistance. Dans la mesure où des tentatives ont été menées en vue de fournir des opportunités d'emploi, elles ont privilégié les initiatives sectorielles traditionnelles afin de réduire le « chômage des jeunes » (souvent, il a surtout été question des jeunes gens). En règle générale de tels programmes accordaient une place importante à la formation professionnelle.

Ces méthodes sont souvent inefficaces, non seulement à cause de leur nature restric- tive, mais aussi parce qu'elles ignorent les réalités de la vie des jeunes. Dans beaucoup de nations en développement, la majorité des filles quittent l'école à l'âge de 10 ou 12 ans et beaucoup d'entre elles travaillent dans le secteur informel. Lorsqu'elles gagnent de l'argent hors du marché de l'emploi salarié, elles pourraient être impliquées dans des activités précaires. Elles n'ont pas accès à l'éducation de base ou à la formation professionnelle et les normes sociales et culturelles immuables peuvent limiter les opportunités qui leur sont offertes dans tous les domaines de la vie. Les conditions de vie des jeunes et leurs besoins en subsistance sont intimement liés. En fait, beaucoup de jeunes filles perçoivent le mariage précoce comme une tentative de leur « employeur » de les faire vivre dans le cadre de la famille austère, dont elles sont tenues de contribuer à pourvoir à leurs besoins - mais sans avoir 
accès aux qualifications de base (dans les pays en développement, il est très rare de trouver une adolescente mariée qui poursuit ses études). Dans certains environnements, les filles, et plus généralement les garçons, quittent la grande famille normale à cause de la pauvreté et finissent par créer leur propre sub-culture à leur intention, un phénomène qui passe souvent inaperçu ou est rejeté catégoriquement étant donné que ces groupes sont perçus comme une menace à la stabilité sociale.

\section{Qu'est-ce qui rend l'approche basée sur les moyens de subsistance}

attrayante lorsque l'on examine la situation des jeunes adolescentes?

L'approche basée sur les moyens de subsistance telle qu'elle est appliquée au spectre de l'âge peut avoir des avantages particuliers par rapport aux jeunes. Les principes qui la sous-tendent ne sont pas nouveaux. Tout d'abord, l'approche fondée sur les moyens de subsistance ne perçoit pas le travail des adolescents comme négatif, au contraire, elle offre une perspective qui permet de considérer le travail comme un moyen de favoriser l'amélioration des compétences chez les adolescents, d'augmenter leurs connaissances grâce à des moyens éducatifs informels ainsi que de renforcer l'estime de soi et la confiance en soi. L'approche basée sur les moyens de subsistance considère le travail comme l'un des nombreux éléments nécessaires au processus de l'épanouissement d'un adolescent de manière efficace.

L'approche fondée sur les moyens de subsistance essaie de comprendre les con-
I Le défi consiste donc à placer solidement les filles sur les rails de l'emploi.

traintes liées à l'économie afin de déterminer les qualifications dont les jeunes ont besoin. Elle tente de lier sous tous ses aspects les facteurs sociaux et économiques qui affectent la vie des jeunes. Dans l'hypothèse la plus optimiste, ces programmes accorderaient de l'attention à la lutte contre la pauvreté (au profit des jeunes et de leurs familles) tout en essayant de créer des opportunités pour ceux qui ne sont pas aptes à trouver un emploi dans le secteur formel, à cause de leur faible niveau d'éducation, de qualification et de ressources. Au départ, un accent particulier porterait sur l'acquisition de compétences plutôt que sur la création d'emplois bien que la finalité fût de trouver un emploi sûr et productif pour les jeunes. L'approche basée sur les moyens de subsistance reconnaît le rôle que le travail joue à plus long terme dans la vie des jeunes et le rôle que les jeunes gens jouent pour leur part dans la vie économique de leur pays ; il ne s'agit pas seulement de trouver un emploi à un moment donné. L'approche essaie aussi de comprendre les besoins des populations catégorielles comme les enfants des rues et les ménages dirigés par des jeunes.

Le développement des moyens de subsistance des adolescents exige que l'on reconnaisse que les adolescents ne forment pas un groupe homogène. Les jeunes 
femmes en particulier ont des besoins spécifiques qui doivent être satisfaits. Dans l'idéal, les opportunités d'emploi et de formation sont offertes dans un contexte sensible à la marginalisation, la mobilité, la culture et aux qualifications des adolescents. Ces programmes s'appuient sur les capacités productives des jeunes et promeuvent des moyens de les améliorer et de les mettre en rapport avec les opportunités d'emploi productif ou d'emploi autonome.

Le travail est considéré non seulement pour les revenus qu'il génère à court terme, mais aussi pour le rôle qu'il joue dans l'épanouissement de soi-même. Comme l'indique la recherche qualitative susmentionnée menée au Bangladesh, en Égypte et en Jordanie, les possibilités de travailler hors du domicile peuvent améliorer le statut des filles au sein de leur famille et de leur communauté, elles renforcent leur estime de soi et développent de futures opportunités au-delà du domaine traditionnel étriqué du mariage et de la procréation. De manière générale, les opportunités de travail pour les filles sont concentrées dans une gamme réduite d'emplois ne nécessitant pas des qualifications poussées et d'accès facile - dont beaucoup sont source d'exploitation - et la ségrégation des sexes sur le marché du travail commence dès le jeune âge. Ainsi, le défi consiste à placer les filles sur une voie professionnelle positive.

\section{Moyens d'existence durables pour les} jeunes: quoi de neuf?

L'approche fondée sur les moyens d'existence durables pour les jeunes est une adaptation du cadre des moyens d'existence durables tel que défini par CARE, le Ministère du développement international du gouvernement britannique et l'Institut d'études sur le développement et elle est de manière plus implicite, en cours d'adoption par la Banque mondiale.

L'objectif de l'approche fondée sur les moyens d'existence durables est d'éliminer la pauvreté. Ses principes fondamentaux — la viabilité et une orientation à plusieurs niveaux axée sur les personnes, dynamique, coopérative et participative menée en partenariat — peuvent être appliqués et explicités pour ce qui concerne les défis spécifiques auxquels les jeunes font face.

Orientation vers les jeunes : l'approche fondée sur les moyens de subsistance admet que les jeunes sont différents des adultes, mais qu'ils sont également différents les uns des autres. Le contexte du mode de subsistance des jeunes adolescentes est par exemple notablement différent de celui des adolescentes plus âgées. Les politiques et interventions doivent reconnaître ce fait et réagir en conséquence.

Dynamisme : l'approche reconnaît les interdépendances entre l'école, le travail, le développement social et la famille. Dans les pays en développement, contrairement à ce que montrent les preuves empiriques, les approches traditionnelles ont dans leur majorité beaucoup insisté sur l'éducation formelle (Graphique 6). L'approche fondée sur les moyens de subsistance n'est pas motivée par des options formelles en matière d’amélioration des compétences et 


\section{GRAPHIQUE 6. Harmonisation des approches fondées su les moyens de subsistance avec des}

facteurs multiples de la vie des adolescents dans les pays en développement

Normes traditionnelles de l'Occident

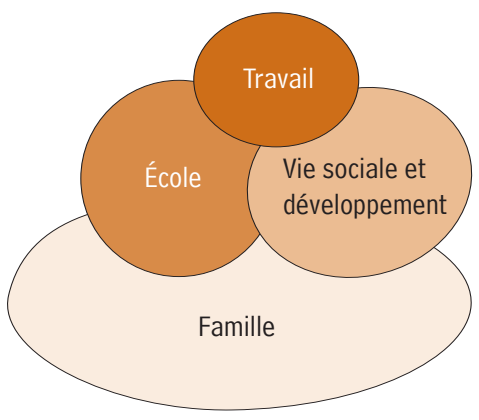

Réalité dans les pays en développement

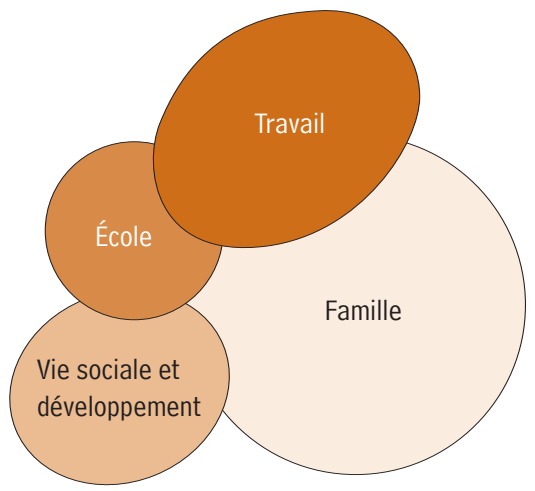

Ce graphique démontre le poids relatif dans la vie des adolescents du travail, de l'école, de la vie sociale de le développement et de la famille. D'après la norme traditionnelle à l'Occident, les adolescents sont d'habitude à l'école et ont une vie sociale assez développée. Les exigences de la famille soutiennent, mais n'empiètent pas sur la vie scolaire ou travailleuse de l'adolescent. Par contre, la réalité dans les pays en développement — à laquelle l'approche fondée sur les moyens de subsistance doit s'adresser — est que les exigences de la famille et du travail se chevauchent et dominent en grande partie la vie adolescente (souvent le travail satisfait les obligations familiales). L'école joue un rôle moins important chez les adolescents parce que l'occasion pour une éducation se présente moins souvent, et quelques-uns peuvent payer l'école seulement en travaillant aussi. La vie sociale, surtout avec des paires, joue un rôle pareil (et pas du tout insignifiant) dans la vie de ces adolescents.

Source : Jamie Schnurr.

comprend que dans bien des cas, le travail, comparé à l'école, occupe une part plus importante du temps des jeunes parce qu'ils doivent travailler à un âge précoce. Cependant, cette exigence peut négativement affecter à court ou à long terme, le développement social d'un jeune et son acquisition de compétences.

L'approche basée sur les moyens de subsistance tient compte des interdépendances à court et à long termes entre les compétences et le développement social d'une part et la capacité actuelle et future de gagner de l'argent d'autre part. Bien que l'idéal fût que les compétences acquises puissent être liées au système formel, l'activité se focalise sur la communauté et pri- vilégie la conception de programmes adaptés aux conditions de vie des adolescentes - par exemple, beaucoup de jeunes femmes travaillent à la maison sous l'autorité d'adultes peu compatissants, sont de nouvelles mariées et vivent dans des ménages où elles sont obligées de rester, sont socialement isolées ou vivent dans des communautés marginales destinées aux jeunes.

Capacité d'adaptation et approche participative : les jeunes sont considérés comme des sujets et non comme des objets. En Afrique, la politique de l'emploi a été fortement politisée par les gouvernements qui tentaient de traiter avec condescendance (et parfois même de réprimer) 
une jeunesse « indisciplinée ». Les programmes n'ont souvent pas reconnu de manière explicite que les jeunes gens étaient en réalité le meilleur atout d'un pays en développement. Ceci est particulièrement manifeste au moment où l'espérance de vie se réduit et la pandémie du VIH fait des ravages au sein de la main d'œuvre instruite. La méthode basée sur les moyens de subsistance, admet que les préférences et les options du groupe de clients, les jeunes dans ce cas, définissent les points d'entrée. La perception que les jeunes ont de leurs capacités et de leurs compétences (qui se sont révélées éclairantes par rapport aux réalités des marchés dans lesquels ils veulent travailler) constituent le fondement de la conception du programme.

Orientation à plusieurs niveaux : l'approche basée sur les moyens de subsistance élabore des programmes selon une démarche ascendante. Les gouvernements et les sociétés ont tendance à orienter l'épanouissement des jeunes vers l'éducation formelle, le sport et les programmes et politiques d'aide sociale à l'enfance. Traditionnellement, ces politiques sont conçues par des représentants au niveau national, qui sont coupés des réalités de la vie quotidienne des jeunes et de leur famille. De plus, ces politiques sont souvent

\section{DÉFINITION DES MOYENS DE SUBSISTANCE}

a définition ad hoc actuelle des moyens de subsistance a évolué par rapport à Lcelle élaborée par Chambers et Conway (1992). Les moyens de subsistance englobent les capacités, les ressources et les opportunités qui permettent de poursuivre les objectifs économiques individuels et ceux du ménage. Les objectifs économiques vont selon une échelle progressive, de la survie à la sécurité à plus long terme pour les futures générations. Différents objectifs impliquent différentes stratégies qui dépendent souvent de différents niveaux de ressources, de différentes vulnérabilités et de différents cycles de vie.

- Les capacités comportent les compétences, une bonne santé, la confiance en soi et l'estime de soi et la capacité à prendre des décisions.

- Les ressources incluent les biens financiers (par exemple, les prêts, l'épargne), les biens matériels (par exemple, le logement, les terrains, les infrastructures) et les biens sociaux (par exemple les liens sociaux et les relations de confiance).

- Les opportunités comprennent les activités de génération de revenus ou d'investissement pour l'acquisition de biens. Les activités pourraient inclure l'emploi autonome, l'emploi salarié, le travail à domicile, la production familiale et l'entretien des relations sociales et communautaires de réciprocité qui créent un capital social. 
élaborées sur la base d'une perception plutôt conceptuelle de ce qui devrait marcher, une approche prétendument déductive. Les moyens d'existence durables utilisent une approche communautaire inductive de l'élaboration des programmes. Les politiques et programmes sont conçus compte tenu des qualifications et options des jeunes et sont, lorsque cela est possible, liés à des institutions formelles à des niveaux plus élevés. Dans le meilleur des cas, ce " cadre » peut et doit être utilisé pour réformer l'éducation traditionnelle et soutenir les programmes et politiques d'aide à l'enfance.

Exécution en partenariat : l'approche basée sur les moyens de subsistance des jeunes est liée aux forces du marché ainsi qu'au secteur privé. Plusieurs grandes sociétés perçoivent les jeunes du secteur informel comme un simple moyen de commercialiser et de distribuer leurs produits. L'approche basée sur les moyens de subsistance des jeunes offre au gouvernement et au secteur privé, la possibilité de travailler ensemble à l'élaboration de programmes et politiques qui tiennent compte de la capacité des jeunes gens et des jeunes femmes, dans le contexte du marché et de leurs stratégies actuelles de subsistance.

Utilisation d'un réseau d'apprentissage :

Réseau du CRDI des moyens de subsistance en Afrique

L'objectif du Réseau du CRDI des moyens de subsistance en Afrique est de contribuer au développement de moyens de subsistance viables grâce à la recherche-action sur des programmes et politiques qui améliorent les capacités et les droits et les relient aux opportunités de subsistance. Le Réseau a pour cible les jeunes gens et les jeunes femmes peu instruits, qui travaillent dans le secteur informel. Alors que de manière générale, l'approche basée sur les moyens de subsistance privilégie les populations marginalisées plutôt que la majorité, en Afrique, la population marginalisée constitue la majorité.

Le Réseau tente aussi de créer des liens entre chercheurs et praticiens afin de générer des connaissances. Il est basé au Centre d'études sur la jeunesse de l'université de Venda, dans la province du Nord en Afrique du Sud. Il a parrainé deux Ph.D. sur les moyens de subsistance des adolescents - les deux étudiants se penchent sur les modèles de subsistance durables pour les jeunes.

Le CRDI a aussi établi son propre programme de travail qui consiste en :

- L'élaboration d'outils et de méthodes d'évaluation des programmes et politiques axés sur les moyens de subsistance ;

- La consolidation et la diffusion des connaissances sur les programmes et politiques qui se sont révélés efficaces ;

- L'élaboration de guides, de trousses d'outils et de modules pour initier et évaluer la réforme des politiques et des programmes; et

- Le développement des capacités et des moyens de tisser des liens entre chercheurs, praticiens et experts. 
L'élaboration des outils et des méthodes s'appuie sur le cadre des moyens de subsistance : d'abord comprendre le contexte des modes de subsistance, puis passer aux programmes et ensuite passer aux politiques. En règle générale, les programmes et les politiques font l'objet d'une évaluation en bloc.

\section{Bien que l'approche basée sur les} moyens de subsistance gagne du terrain, elle n'a pas encore pleinement été mise en œuvre

Face à ces politiques et programmes, les gouvernements ainsi que les bailleurs de fonds ont jusque-là réagi au gré des circonstances. La mise en œuvre des programmes de moyens de subsistance est généralement limitée à cause du manque de cohérence entre les principaux acteurs chargés de la conception et de la mise en œuvre des politiques et programmes. Par exemple, dans nombre de pays d'Afrique subsaharienne, il pourrait exister des politiques officielles de jeunesse ou des politiques officielles axées sur les moyens de subsistance. Il pourrait également y avoir des activités adéquates menées par des entités non gouvernementales. Il est cependant rare de voir les deux entités travailler de concert. Ce manque de coordination entre politiques et programmes est préoccupant. Par exemple le Malawi ainsi que la Zambie ont formulé des politiques de jeunesse, cependant, les gouvernements de ces deux pays ne les mettent pas en œuvre faute de ressources. Un certain nombre d'ONG internationales et locales (par exemple CARE et le Forum des dirigeants d'entreprises zambiens) exécutent toutefois des programmes dont les objectifs sont en principe conformes aux politiques mises en place. Il s'ensuit que les politiques sont mises en œuvre de facto mais il n'existe pratiquement pas de communication entre les concepteurs des politiques (des représentants du gouvernement) et les personnes chargées de la mise en application de facto (bailleurs de fonds et ONG).

En outre, le Sommet sur le microcrédit qui s'est tenu en février 1997 à Washington D.C. a eu pour effet d'attirer l'attention sur le microcrédit comme remède universel en dépit du fait qu’il existe en règle générale, une faible compréhension de ce qui marche et ce qui ne marche pas, notamment lorsque de jeunes adultes sont concernés. Dans certains cas, les programmes de crédit ont été mis en œuvre dans les pays en développement en guise de cadeau aux jeunes en échange de leur soutien politique présumé, alors que peu d'attention est accordé au processus de mise en application ou aux résultats. Ceci a entraîné l'impossibilité de développer de nouvelles compétences pour les jeunes et d'assurer les capacités d'amélioration des compétences et de subsistance par l'intermédiaire des programmes de microcrédit et d'épargne. Par exemple, nombre de pays d'Afrique subsaharienne ont initié des programmes de microcrédit afin de dynamiser l'emploi autonome parmi les jeunes gens et les jeunes femmes. Les programmes de crédit 
financés et contrôlés par les gouvernements nécessitaient au départ deux semaines de formation en acquisition de compétences en affaires. Les jeunes se sont mobilisés et ont protesté contre l'obligation de suivre une formation de deux semaines alors que les participants à d'autres programmes de microcrédit avaient reçu le crédit sans aucune formation. À la suite de ces protestations, la formation a été réduite à deux jours. Les gouvernements ont rapidement réagi à cause de la nature hautement politique de leurs relations avec les jeunes. Les programmes de crédit ont finalement connu un échec et ont eu pour conséquence un taux élevé de défauts de remboursement et un sentiment d'échec parmi les jeunes gens et les jeunes femmes. Quelques conclusions peuvent cependant être tirées : (1) les programmes de crédit ne sont pas nécessaires pour tous les jeunes; (2) les programmes de crédit ne devraient pas être gérés par les gouvernements, mais plutôt par un consortium d'agences de formation et d'évaluation du crédit ; (3) le mentorat est crucial pour la réussite des programmes de crédit et (4) la mise en place des critères de sélection d'objectifs et de rendement est indispensable.

Le scepticisme est persistant dans certains milieux. Ceux qui s'opposent au travail de tous les enfants sauf des adolescents les plus âgés, craignent que les adolescents ne soient vulnérables à l'exploitation au travail, que le travail ne détourne l'attention des adolescents de l'école, qu'il freine leur développement physique et psychologique et que les jeunes travailleurs plus faiblement rémunérés ne « volent » les emplois des premiers titulaires de revenus. (Les arguments portant sur le vol d'emplois reprennent ceux d'il y a 25 ans, lorsque l'attention portait sur l'accès des femmes aux compétences et à l'emploi.) Tous ces arguments ont quelque valeur, cependant, les opportunités accrues de subsistance ne semblent pas avoir les effets néfastes décrits ci-dessus et, en réalité, la synergie est permise. L'objectif est d'offrir une série d'opportunités qui renforcent les capacités sociales et économiques de base des jeunes.

\section{À ce jour quelle est l'expérience en} matière de création et/ou de soutien des moyens de subsistance ? ${ }^{15}$

\section{Synthèse des fondements actuels et potentiels des programmes de subsistance des adolescents}

L'approche basée sur les moyens de subsistance est de plus en plus admise au sein de la communauté de développement et est présentement utilisée comme outil de programmation par nombre d'organismes de développement. L'un des éléments qui attirent dans cette approche concerne le fait qu'elle soit axée sur les personnes. Elle pri-vilégie les objectifs économiques des individus et des ménages et est en mesure de prendre en compte les dynamiques et les interactions complexes au fil du temps. Elle tient compte des capacités et des ressources individuelles ainsi que des structures grâce auxquelles les gens peuvent chercher à atteindre leurs objectifs économiques. 


\section{Domains d'action}

Les moyens de subsistance peuvent être définis comme les capacités, les ressources, et les opportunités qui permettent à un individu d'atteindre ses objectifs économiques. Trois domaines d'actions peuvent être pris en considération compte tenu de ces définitions.

Les capacités. Les programmes qui développent les capacités de subsistance pourraient mettre l'accent sur les capacités de lecture et d'écriture et au calcul, les compétences professionnelles les compétences en affaires et en gestion du budget, les connaissances spécialisées, le développement de l'esprit d'entreprise et la formation en dynamique de la vie. L'acquisition de l'estime de soi et de la confiance en soi ainsi que la garantie contre la violence en vue de chercher à atteindre les objectifs économiques peuvent aussi être considérées comme faisant partie des capacités de subsistance.

Les ressources. Le deuxième domaine d'action relatif aux moyens de subsistance inclut les programmes qui facilitent l'accès aux ressources et leur contrôle. Les programmes de microfinance qui sont peutêtre les plus courants dans ce domaine, développent l'accès aux ressources financières par le biais de la mise en place de services de crédit et d'épargne et dans quelques programmes, par l'intermédiaire des services d'assurances. Des exemples d'autres programmes orientés vers les ressources incluent ceux qui introduisent de nouvelles technologies ou qui mettent l'accent sur un meilleur accès aux ressources matérielles comme les terrains et les biens de production et sur leur meilleur contrôle.

Les opportunités. Le troisième domaine d'action inclut des programmes qui structurent les opportunités. Ils peuvent être classés en cinq domaines majeurs : (1) les emplois, au nombre desquels les projets générateurs de revenus, les programmes de travaux publics, le développement d'entreprises coopératives et le développement de petites et moyennes entreprises créatrices d'emplois pour les adolescents ; (2) la promotion de l'accès aux marchés, des infrastructures, des services, et des opportunités d'emploi ; (3) la protection et la promotion des droits, y compris les droits de propriété, les droits des travailleurs, les droits à un salaire égal, les droits à la représentation; (4) la mise en place d'institutions telles que les organisations intermédiaires, les associations d'employés, les organisations de femmes et les alliances institutionnelles stratégiques qui préconisent la promotion du droit et la sécurité des environnements de travail ou qui mettent en place des réseaux de soutien social et/ou professionnel et (5) les programmes qui œuvrent pour les changements structurels nécessaires à la création d'opportunités de revenus pour les groupes économiquement défavorisés, y compris les changements d'orientation, de législation, de réglementation et de normes sociales.

La synergie entre ces trois domaines d'action est très importante. Les capacités 
et les ressources sont nécessaires si l'on veut tirer parti des opportunités et vice versa. Cependant, les programmes individuels ne doivent pas nécessairement inclure des volets de tous les trois domaines, notamment parce que les approches intégrées peuvent être trop compliquées pour fonctionner de manière adéquate ou pour toucher un nombre important de personnes et elles pourraient être trop onéreuses. Le cadre des moyens de subsistance suggère cependant une manière d'aborder un contexte particulier ou un groupe cible et il aide à prendre en considération différentes options de programmation. Il agrandit l'objectif qui permet de prendre en compte une vaste gamme de programmes destinés aux adolescentes, qui vont bien au-delà du crédit (Tableau 1).

Le cadre des moyens de subsistance permet de mieux comprendre des domaines où il existe une multitude de programmes et les domaines où ils font défaut. Les programmes sur les moyens de subsistance des femmes, tendent par exemple à consacrer leur énergie à fournir une formation en gestion financière et axée sur les compétences, alors que très peu offrent des opportunités. Le cadre aide aussi à identifier les domaines où les programmes ont échoué. La création d'emplois en est un exemple ; les programmes de génération de revenus et de travaux publics n'ont souvent pas atteint cet objectif à long terme. Le cadre offre aussi un point de départ pour la réflexion sur les programmes par rapport aux objectifs de renforcement des capacités en matière de subsistance, le développement de l'accès aux ressources et leur contrôle ainsi que la structuration des opportunités qui permettent aux individus de poursuivre leurs ambitions économiques.

\section{Quelles sont les possibilités}

d'implication des adolescents dans les programmes de microfinance?

\section{Dans quelles circonstances cela} peut-il se faire correctement?

Il existe 7000 à 10000 programmes de microfinance en cours d'exécution à travers le monde, et en conséquence, il existe plusieurs approches et modèles différents d'attribution de microfinance. Les programmes vont selon une échelle progressive, de modèles minimalistes qui se focalisent principalement sur les objectifs financiers et institutionnels - comme le ciblage d'un nombre important de clients avec une gamme réduite et standardisée de services financiers — à des modèles intégrés de « crédit-plus » - des programmes qui ont souvent des objectifs de développement diversifié (comme la réduction de la pauvreté ou l'autonomisation des femmes) et offrent plus que de simples services financiers. En réalité, la plupart des programmes sont hybrides. Cependant, l'expérience relative aux programmes destinés aux jeunes est limitée, en ce sens que les programmes ne tiennent généralement pas compte du contexte dans lequel les adolescents vivent à divers endroits et dans diverses conditions, n'expérimentent pas les approches novatrices de mise à disposition de produits et services financiers au profit des adolescents. 
TABLEAU 1. Cadre de programmation des moyens de subsistance

$\begin{array}{ll}\begin{array}{l}\text { Objectifs du } \\ \text { programme }\end{array} & \begin{array}{l}\text { Exemples concrets (pourraient } \\ \text { ne pas nécessairement } \\ \text { impliquer des adolescents) }\end{array}\end{array}$

LES PROGRAMMES QUI DÉVELOPPENT LES CAPACITÉS

Développe les

compétences

- Services de formation (formation axée sur les compétences, formation axée sur les activitiés, formation en gestion, autres types de formation pour la mise en valeur des ressources humaines)

- Développement de l'entrepreneurial

Rendre les femmes autonomes

- Organisation de groupes et autres stratégies d'intermédiation sociale

- Développement de l'esprit d'initiative

- Formation à une meilleure connaissance de la législation
- Association des femmes autonomes (SEWA), Lucknow

- Centre international pour l'entrepreneuriat et le développement professionnel/Inde

- Syndicat de la SEWA

- Comité du Bangladesh pour l'essor rural (BRAC)

\section{LES PROGRAMMES QUI DÉVELOPPENT LES RESSOURCES}

\begin{tabular}{|c|c|}
\hline $\begin{array}{l}\text { Fournir des services } \\
\text { financiers }\end{array}$ & $\begin{array}{l}\text { - Crédit } \\
\text { - Épargne } \\
\text { - Autres services financiers (par exemple, } \\
\text { transfert des paiements automatiques, } \\
\text { assurances) }\end{array}$ \\
\hline $\begin{array}{l}\text { Améliorer l'accès aux } \\
\text { ressources non } \\
\text { financières }\end{array}$ & $\begin{array}{l}\text { - Réforme agraire et droits de propriété } \\
\text { - Programmes de ressources des } \\
\text { biens communaux } \\
\text { - Développement technologique }\end{array}$ \\
\hline
\end{tabular}

\section{LES PROGRAMMES QUI STRUCTURENT LES OPPORTUNITÉS}

\section{Créer des emplois - Projets générateurs de revenus \\ - Coopératives \\ - Autres entreprises de groupe \\ - Développement de petites entreprises qui créent des emplois pour les jeunes \\ - Titan (une compagnie de fabrication de montres) \\ - Association pour la protection de l'environnement, Maqattam, Le Caire}

Promouvoir l'accès aux
marchés, aux terrains, aux
services, aux infrastructures

Protéger et promouvoir les droits de propriété, les droits de travailleurs, les droits à un traitement adéquat, les droits à la représentation

- Programmes de liaisons entre les marchés

- Organisation par le truchement des syndicats, des associations d'employés, des associations de jeunes

- Formation en sensibilisation aux lois et aux droits

- Programmes de protection sociale destinés aux employés

Mettre en place des institutions

Promouvoir des changements structurels (législation, politiques, normes sociales)
- Soutien financier, soutien à la gestion et formation du personnel pour les organisations qui œuvrent pour développer de nouvelles opportunités pour les jeunes

- Réforme juridique

- Réformes stratégique

- Initiatives pour modifier les normes sociales
- Banque du Kenya Rural Enterprise Program (K-REP)

- La Banque Grameen

- Banque de la SEWA

- Centre pour l'éducation des masses et la science (CMES)
- Programmes sectoriels du BRAC

- Jeunesse ouvrière chrétienne (Belgique)

- Campagne de la SEWA pour les femmes autonomes du secteur informel

- Programmes de formation de ADITHI en sensibilisation au droit traditionnel et au droit

\footnotetext{
- Formation en autonomisation par le CMES - SEWA

- La Banque Grameen

- ADITHI
} 
Sur la base des expériences que les femmes mariées ont acquises avec les programmes de crédit et d'épargne, les résultats potentiels d'un modèle de « créditplus » destiné aux adolescentes pourraient être significatifs. Les groupes affinitaires associés aux mécanismes d'emprunt et d'épargne peuvent contribuer à créer des réseaux sociaux et à fournir des informations et une formation dans d'autres domaines que le crédit. Dans les sociétés où les filles sont souvent isolées et vulnérables, ce type de soutien social peut être particulièrement inestimable et pourrait même faciliter la transition vers le mariage.

\section{Les adolescentes ont-elles accès au} crédit ? Le cas du Bangladesh ${ }^{16}$

Le microcrédit est devenu la plus importante source d'emprunt formel dans les zones rurales du Bangladesh et représente environ deux tiers de l'ensemble du crédit institutionnel disponible dans ces zones. Plus de 1000 ONG sont impliquées dans des activités de microcrédit au Bangladesh et leurs prêts sont principalement destinés aux paysans sans terre et aux femmes. Pris ensemble, les programmes de microcrédit des ONG et du gouvernement prêtent de l'argent à au moins 10 millions d'individus au Bangladesh.

Un échantillon des établissements de crédit au Bangladesh a fait l'objet d'une enquête afin de déterminer comment elles traitent avec les adolescentes, mariées ou non. Cela comprenait un examen du BRAC, de l'Association pour la promotion sociale
(ASA), de la Banque Grameen, du Centre pour l'éducation des masses et la science (CMES) et de deux programmes de l'Office du développement rural du Bangladesh (BRDB) - le Projet de développement rural 12 (RD-12) et le Programme intégré d'aide à la promotion des femmes en milieu rural (IRWDP). Chaque établissement dispose de critères que les bénéficiaires doivent remplir pour être éligibles de même que des lois et règlements auxquels les bénéficiaires de prêt sont tenus de se conformer (Tableau 2).

Le BRAC encourage la participation des adolescentes à plusieurs de ses activités, au nombre desquelles les programmes d'éducation formelle et non formelle, l'éducation juridique et l'éducation à la sensibilisation au droit, et des programmes de soins de santé et d'éducation sanitaire. Avec la microfinance, le BRAC préfère faire participer des femmes mariées selon le principe que les femmes célibataires se déplacent après leur mariage. Le BRAC exclut à dessein les filles célibataires de ses programmes de crédit afin de maintenir les taux de remboursement à des niveaux aussi élevés que possible. En outre, beaucoup d'agents de crédit sont des hommes, ce qui pose un problème culturel en termes d'accès aux femmes non mariées.

De même, I'ASA préfère ne pas accorder de crédit aux adolescentes non mariées pour les mêmes raisons que le BRAC : une fois mariées, il sera difficile de recouvrer une dette active. Les adoles- 
TABLEAU 2. Critères que les bénéficiaires doivent remplir pour pouvoir prétendre à un prêt

\begin{tabular}{|c|c|}
\hline $\begin{array}{l}\text { BRAC } \\
\text { 1. Travailler comme manœuvre environ } \\
100 \text { jours par an } \\
\text { 2. Posséder moins d'un demi-arpent de } \\
\text { terre } \\
\text { 3. Être âgée de } 18 \text { à } 55 \text { ans } \\
\text { 4. Seules les femmes peuvent en bénéficier } \\
\text { 5. Être physiquement active } \\
\text { 6. Être une résidente permanente de la } \\
\text { localité } \\
\text { 7. Ne pas avoir de source de revenus } \\
\text { réguliers } \\
\text { 8. Ne pas être membre d'une autre } \\
\text { organisation } \\
\text { ASA } \\
\text { 1. Pauvres pauvres, principalement les } \\
\text { femmes depuis } 1985 \\
\text { 2. Être un résident permanent de la loca- } \\
\text { lité } \\
\text { 3. Être âgé de } 18 \text { à } 50 \text { ans } \\
\text { 4. Les revenus du ménage ne doivent pas } \\
\text { dépasser } 1200 T K \text { ( } 24 \$ \text { USD) par mois } \\
\text { 5. Travailler comme manœuvre six mois } \\
\text { par an } \\
\text { 6. Posséder un demi-arpent de terre ou } \\
\text { moins } \\
\text { 7. Être mentalement et physiquement } \\
\text { capable de mener des activités généra- } \\
\text { trices de revenus } \\
\text { 8. N'être ni étudiant, ni mendiant } \\
\text { 9. Être marié ; cependant, les femmes } \\
\text { divorcées, séparées ou veuves sont } \\
\text { acceptées en fonction de leur accep- } \\
\text { tabilité sociale passée }\end{array}$ & $\begin{array}{l}\text { LA BANQUE GRAMEEN } \\
\text { 1. Les pauvres (démunis) } \\
\text { 2. Les groupes de paysans sans terre } \\
\text { constituent l'unité principale et fonda- } \\
\text { mentale de la structure } \\
\text { 3. Posséder moins d'un demi-arpent de } \\
\text { terre } \\
\text { 4. Groupe composé d'au moins } 5 \text { à } 10 \\
\text { personnes aux vues similaires et de } \\
\text { condition économique semblable } \\
\text { 5. Avoir des biens dont la valeur est } \\
\text { inférieure à la valeur d'un arpent de } \\
\text { terre de qualité moyenne } \\
\text { CMES } \\
\text { 1. Être membre du système } \\
\text { d'enseignement élémentaire du CMES } \\
\text { 2. Avoir au moins suivi les deux pre- } \\
\text { mières années d'éducation élémentaire } \\
\text { 3. Être âgé de } 11 \text { à } 18 \text { ans } \\
\text { 4. Ne pas avoir de liens de parenté avec } \\
\text { les autres membres du groupe } \\
\text { BRDB/RD-12 } \\
\text { 1. Posséder moins d'un demi-arpent de } \\
\text { terre } \\
\text { 2. Être âgé de } 18 \text { à } 50 \text { ans } \\
\text { 3. Être un résident permanent de la localité } \\
\text { 4. Ne pas avoir de source de revenus } \\
\text { réguliers } \\
\text { 5. Ne pas être membre d'une organisa- } \\
\text { tion, quelle qu'elle soit } \\
\text { 6. Avoir une domicile fixe } \\
\text { BRDB/IRWDP } \\
\text { 1. Femmes âgées de } 18 \text { à } 35 \text { ans } \\
\text { 2. Être disposée à participer aux activités } \\
\text { de programme destiné aux femmes }\end{array}$ \\
\hline
\end{tabular}

centes non mariées sont considérées comme étant immatures et cela constitue pour le prêteur, un souci supplémentaire au regard du remboursement. Cependant, les adolescentes mariées sont prises en compte dans les groupes ASA réguliers. L'ASA fixe l'âge minimum auquel on peut bénéficier d'un prêt à 18 ans, mais cette exigence fait l'objet de dérogation pour les jeunes filles mariées.

La Banque Grameen, une institution connue pour son rôle de pionnière en microfinance, est ouverte à l'octroi des prêts aux adolescents. Elle est d'avis que beaucoup de familles monoparentales ont besoin d'une source de revenus supplé- 
mentaire pour survivre et les adolescents peuvent souvent aider à remplir ce rôle. La majorité des adolescents - les garçons comme les filles - seront un jour, responsables d'un ménage eux-mêmes et ainsi, apprendre à gagner et à gérer des revenus constitue une importante dynamique de la vie. Grameen ne s'oppose pas à l'octroi de crédits en faveur des jeunes de moins de 18 ans pour les raisons ci-dessus. Récemment, la banque a initié un programme expérimental de crédit pour l'éducation des garçons et des filles de ses emprunteurs. Le programme fournit des prêts pour s'acquitter des frais de scolarité, qui devront être remboursés lorsque l'étudiant aura eu ses diplômes et commencé à générer des revenus.

Créé en 1991, le Centre pour l'éducation des masses et la science ${ }^{17}$ offre une éducation non traditionnelle, une formation axée sur les compétences et octroie un crédit à 20000 jeunes filles et garçons du Bangladesh. Considérant que les filles qui ont quitté l'école ont encore besoin d'éducation, particulièrement de cette éducation qui peut améliorer les perspectives d'emploi, le CMES a élaboré un programme qui intègre l'apprentissage et le travail dans le cadre d'une initiative de plus grande envergure afin de donner aux filles des possibilités d'épanouissement et de développement personnels.

Les filles sont habilitées à participer aux divers programmes du CMES si elles ne vont pas à l'école et si elles sont célibataires. Bien qu'à ses débuts le CMES ne fût pas une agence octroyant un crédit aux filles, il a vite décidé que la capacité à gagner de l'argent — même de façon modeste - est partie intégrante de l'adolescence. Malgré les avertissements des établissements de crédit majeurs qui affirmaient que les crédits aux jeunes filles célibataires ne pouvaient prospérer, le CMES a commencé à en accorder à cette frange de la population. Bien que l'organisation ait connu certains problèmes - leur défi majeur étant de s'assurer que les bénéficiaires de prêts gardent le contrôle de leurs fonds plutôt que de les donner à un parent ou un frère - elle a aussi développé la formation en vue d'essayer d'encourager les filles à s'engager dans des emplois très peu traditionnels comme ceux de propriétaire d'épicerie, gérante de poussepousse, de blanchisseuse professionnelle et de photographe. Le CMES s'est rendu compte que les filles des familles les plus pauvres sont les moins susceptibles de faire l'expérience des emplois non traditionnels. À ce jour, les diplômées qui ont le mieux réussi ont monté leurs propres affaires dans le secteur de la confection.

\section{Perception que l'on a des filles en tant qu'emprunteuses potentielles}

Ainsi qu'on l'a noté plus haut, les adolescentes célibataires sont perçues comme constituant un groupe à haut risque en matière de prêt, à cause de leurs tendances migratoires dues au mariage. Lorsqu'une fille se marie, la plupart des décisions sont prises par la famille du mari et cela est perçu comme un risque supplé- 
U ne étude de certains programmes actuels de crédit en Inde et au Bangladesh suscite d'importantes questions au sujet des adolescentes, des gains et du mariage :

- Les filles partent-elles avec leur épargne lorsqu'elles se marient?

- Sont-elles en mesure de conserver les biens qu'elles ont acquis ?

- Leurs biens sont-ils laissés au domicile familial comme assurance en cas de problèmes au domicile conjugal ?

- Ces biens peuvent-ils être utilisés comme outils de négociation dans leur domicile conjugal ?

mentaire. En outre, les jeunes filles célibataires sont considérées comme étant trop immatures pour se conformer aux modalités de remboursement quelles qu'elles soient. Cependant les filles mariées âgées de 18 ans ou moins peuvent être incluses dans le programme de prêt de l'établissement de crédit, parce que le mariage est perçu comme le rite du passage à la maturité et à l'âge adulte.

Beaucoup de représentants qui ont été interrogés dans le cadre de cette étude ont reconnu que les adolescentes pourraient aussi vouloir accéder au crédit. Un programme conçu pour des filles célibataires pourrait avoir une structure différente de celle destinée aux femmes mariées. Il inclura notamment des mécanismes de « crédit-plus » et bénéficierait d'un suivi rigoureux, d'une supervision et de la formation en création de petites entreprises et en production.

\section{Quels types de programmes de} subsistance existe-t-il pour les filles en Inde ? 18

Une gamme de programmes de subsistance au profit des adolescentes en Inde met l'accent sur la formation professionnelle et celle axée sur les compétences bien que leur portée et leur pérennité semblent limitées. Dans leur majorité, ils incluent des jeunes filles célibataires ou nouvellement mariées qui n'ont pas encore quitté le domicile familial.

Un programme exécuté par ADITHI travaille de manière plus spécifique avec des filles de Bihar, âgées de 8 à 14 ans. Les filles reçoivent des chèvres et on leur apprend à les élever, à les accoupler et à les vendre dans le cadre d'un programme d'épargne. Les bénéfices permettent aux filles de commencer à se doter de biens. C'est cet aspect qui différencie ce programme en ce sens qu'il est l'un des rares à encourager l'épargne. Dans certains cas, la capacité d'épargne des filles est telle que les parents demandent à leur emprunter de l'argent ! D'autres filles utilisent leur épargne pour poursuivre leurs études ou pour acheter des bijoux. L'épargne est importante pour les adolescentes parce c'est un moyen d'accumuler des ressources pour une utilisation future dont elles auront la maîtrise.

Un autre programme exécuté par le MYRADA est lié à l'un des principaux fabri- 
cants de montres en Inde. Les filles célibataires âgées de 16 à 20 ans achèvent un programme de formation qui leur enseigne la gestion financière et la planification stratégique. Elles travaillent ensuite dans une coopérative indépendante pour produire des maillons de bracelet et les fournir à l'usine ; au cours du processus, elles acquièrent des compétences, gagnent de l'argent et épargnent.

Les adolescentes indiennes ont-elles accès au crédit ?

Actuellement, il existe peu d'opportunités de crédit et d'épargne pour les adolescentes en Inde, même si une structure dynamique de promotion des moyens de subsistance existe pour les femmes plus âgées (souvent mariées). Outre l'épargne et le crédit, ceci comprend des services de soutien social comme les services de santé et l'alphabétisation; des initiatives de lutte contre la pauvreté, qui promeuvent l'accès aux marchés et des organisations qui essaient d'améliorer les infrastructures, de promouvoir les droits et de faciliter l'accès à la propriété.

Il ne semble pas y avoir de programmes de crédit qui cible les adolescentes ou de programmes octroyant des crédits au profit des adolescents. Cependant, au cours des discussions avec les participantes et les organisatrices de l'association des femmes autonomes (SEWA), il est apparu que les filles sont en réalité celles qui profitent de la participation de leurs mères aux programmes de crédit et d'épargne. Par exemple, Iorsqu'une fille voit sa mère gagner de l'ar- gent, elle se rend compte qu'une femme peut diriger une affaire et exercer des activités publiques. De plus, les activités génératrices de revenus des femmes offrent à leurs filles plus d'opportunités d'avoir accès à l'éducation et aux soins de santé.

\section{Un projet pilote destiné à accorder le} crédit aux adolescentes à Nairobi ${ }^{19}$

Le Population Council, en collaboration avec le Kenya Rural Enterprise Program (K-REP, une organisation de premier plan en matière de microfinance au Kenya), a initié un projet destiné aux jeunes femmes célibataires de Nairobi. Le projet, appelé TRY (Tap and Reposition Youth), étudie le rôle de l'épargne et du crédit dans la vie des jeunes femmes. Les membres du personnel du Council espèrent mieux comprendre les incidences du crédit et de l'épargne sur ce groupe d'âge. Par exemple, ils espèrent apprendre si le crédit et l'épargne renforcent les options économiques des jeunes femmes et si leur expérience de la gestion d'une affaire peut changer l'autoperception d'une femme et ses relations avec les autres. Pour le K-REP, le projet TRY offre la possibilité d'étudier la faisabilité des prêts aux plus jeunes qui utilisent leurs services. Ils espèrent découvrir si les adolescents, les adolescentes en particulier, sont des clients fiables en matière de microfinance.

\section{Qui sont les clientes?}

Les participantes au projet TRY sont âgées de 16 à 24 ans. Elles sont soit diplômées d'une école, soit elles ont abandonné leurs études, elles vivent dans un bidonville de 
Nairobi et sont présentement sans emploi. Certaines ont une expérience des affaires ; toutes veulent faire carrière dans les affaires. La première cohorte de prêt est un mélange de femmes mariées et célibataires, dont certaines ont déjà des enfants.

\section{Comment le TRY fonctionne-t-il ?}

Les premiers prêts ne dépassent pas 200 USD ; les participantes rembourseront les crédits avec un taux d'intérêt annuel de 15 pour cent, qui est légèrement inférieur au taux d'intérêt débiteur actuellement pratiqué par les banques commerciales au Kenya. Le projet TRY offre des services financiers et non financiers aux participantes. Les cinq volets majeurs de leurs services sont la mobilisation de l'épargne, les prêts, une formation complémentaire en gestion du crédit, une formation de base en gestion des entreprises (y compris la comptabilité) et la préparation à la vie quotidienne. La dernière catégorie inclut les immobilisations incorporelles comme la prise de décision, les qualités de chef, l'assertivité et la prise de conscience des rôles de genre.

Le modèle d'octroi du crédit pour le projet TRY est très proche du modèle standard du K-REP, qui s'inspire lui-même du modèle de la Banque Grameen. Le projet utilise des mécanismes de prêts basés sur les groupes et garantis par ces derniers; les membres du groupe garantissent mutuellement les prêts à la place des biens matériels affectés en garantie. Chaque groupe détermine ses propres règles de participation. Le groupe tient des réunions hebdomadaires qui sont l'occasion de rembourser, de solliciter le soutien du groupe et d'offrir divers services non financiers. En outre, chaque participante doit épargner chaque semaine.

Le Population Council et K-REP ont commencé à inscrire les participantes en septembre 1998. À partir d'octobre 1999, il y en avait 105. Toutes ont bénéficié d'une semaine de formation intensive en gestion des entreprises avant le versement des prêts en juin 1999. Pour l'instant, 90 jeunes femmes ont reçu des prêts. Environ 9 ont abandonné le projet, certaines de leur plein gré d'autres parce qu'elles y ont été contraintes. Certaines ont des maris qui leur ont défendu d'y participer, quelques-unes ont parlé de certains aspects du projet qui allaient à l'encontre de leurs principes religieux et d'autres ont été jugées peu fiables et ont dû quitter à la demande de leurs homologues. Celles qui sont restées ont en moyenne, épargné l'équivalent de 7 USD grâce à diverses activités dont la coiffure, la construction de maisons, la couture, la vente de produits d'épicerie et la vente de vêtements d'occasion. Les taux de remboursement après un an étaient de 70 pour cent.

\section{Informations sur la faisabilité} du projet pilote TRY

Le projet pilote offre une occasion unique de mener des recherches. Le Population Council étaie soigneusement tous les aspects du projet TRY, y compris les enquêtes initiales et finales, les études de 
cas longitudinales, les groupes de discussion thématique et le suivi des abandons. Le Council et le K-REP essaient de déterminer si un projet de crédit adéquatement conçu et mis en œuvre à l'intention des adolescentes, constitue une option viable dans la région parce que le Kenya a connu quelques expériences en matière d'épargne et de crédit, dont beaucoup semblent avoir échoué. Des études d'impact peuvent être menées à une date ultérieure ; pour l'instant, les chercheurs essaient de déterminer si un tel projet peut fonctionner correctement et tirent les leçons de l'expérience.

Les chercheurs du Council suivent plusieurs variables de manière longitudinale. Ils cherchent à mieux comprendre comment les participantes au projet TRY utilisent leur temps, ce qu'elles gagnent, comment elles dépensent et épargnent. Ils veulent comprendre leurs habitudes en matière de remboursement de prêts, les attitudes de genre et les rapports de pouvoir entre elles ainsi qu'avec les personnes qui comptent pour elles. Ils mènent aussi des études de cas afin de corroborer les expériences de quelques jeunes femmes qui sont représentatives de différentes expériences en matière de profil démographique et d'exploitation d'une entreprise. De plus, ils dirigent des groupes de discussion thématique à des étapes clés du projet TRY — juste avant que les filles ne reçoivent les prêts, juste après qu'elles les ont reçus et lorsqu'un groupe a remboursé ses prêts. Les abandons font aussi l'objet d'un suivi parce qu'il est important de déterminer pourquoi les jeunes femmes quittent le programme. Grâce aux activités de recueil de documents à long terme, les chercheurs du Council étudient des éléments qui vont au-delà des taux de remboursement. Par exemple ils essaient de déterminer si l'autoperception des filles est différente. S’ils parviennent à établir la preuve de ce changement, ils seront mieux à même de comprendre si le crédit constitue un fardeau ou une opportunité pour les jeunes femmes.

\section{Enseignements}

Le projet TRY en est encore à ses débuts, mais les chercheurs du Council ont jusque-là appris un certain nombre de choses. D’abord, le K-REP a de l'expérience (il a été créé en 1984) et il fait preuve de professionnalisme et il s'est donc révélé être un excellent partenaire. Le modèle de prêt basé sur les groupes semble être approprié pour le projet TRY et les groupes unisexes semblent être d'une importance ca-pitale. Les groupes prennent leur vie en charge et mettent en place des réseaux sociaux pour les filles, en plus des opportunités de crédit et d'épargne. Chaque groupe a mis au point son propre règlement; les membres le suivent mais font parfois plus que ce qui est requis. Par exemple lorsqu'une jeune femme a un bébé, les autres membres du groupe apportent leur aide et leur soutien à la nouvelle mère. Les groupes satisfont d'autres besoins dans la vie des participantes et cela contribue à améliorer le programme. 
En outre, le volet formation semble être très utile pour les participantes. L'apprentissage de l'épargne et ensuite l'accumulation d'une modeste épargne permettent aux participantes du projet TRY de faire des prévisions et d'avoir des projets d'avenir.

Les membres du personnel du K-REP affirment que les cinq premiers mois au cours desquels ils prêtent aux adolescentes se sont passés sans accroc. Ils ont le sentiment que la conception du projet est d'une grande portée pour s'adresser à un groupe particulier, de même que l'ensemble des services offerts. De plus, ils ont appris qu'ils doivent prendre en considération la recevabilité d'une adolescente à un prêt dans le contexte de sa famille et des structures d'assistance, contrairement à ce qui se fait pour les clientes les plus âgées.

\section{Quels sont les éléments d'une formation appropriée en matière de création d'entreprise ?20}

Les programmes d'enseignement et de formation professionnels constituent deux domaines qui permettent aux adolescents des deux sexes d'acquérir les qualifications qu'ils peuvent utiliser pour développer des moyens de subsistance. Étant donné le coût et le temps nécessaire à l'organisation et à la participation à des programmes de formation, ces derniers doivent enseigner des compétences pratiques et utiles sur le marché du travail. Un bon programme de formation tient compte de l'environnement économique général dans lequel le sta- giaire utilisera ses qualifications et autant que possible, il évite de cantonner les filles dans des emplois mal rémunérés traditionnellement dominés par les filles.

\section{Principes directeurs d'une formation} en gestion d'entreprise

- Tenter de former les individus à travailler dans les nouveaux domaines de croissance suscités par la demande ; se méfier des formations qui préparent les stagiaires à travailler dans des domaines déjà saturés.

- S'assurer que les qualifications sont adaptées aux besoins de la communauté. Les consommateurs auront-ils les moyens d'acheter les produits ? Les qualifications adéquates dans un environnement urbain pourraient ne pas être utiles dans les zones rurales.

- Lorsque l'on travaille avec les filles et les femmes, le fait de les encourager à s'engager dans les secteurs traditionnellement « masculins » peut être moins productif que de les former pour un nouveau secteur en cours de croissance encore " exempt de discrimination sexuelle ».

- S'assurer que les programmes demeurent simples et cohérents. Un ou deux objectifs pourraient suffire ; les bons programmes donnent toujours des résultats. Par exemple, un programme axé sur l'acquisition de compétences et qui tente d'améliorer les connaissances des stagiaires en matière de santé et d'hygiène de vie, 
est moins susceptible d'atteindre ses objectifs qu'un programme qui se concentre sur une bonne formation.

- Exploiter les connaissances traditionnelles, mais considérer les barrières traditionnelles avec circonspection.

- Ne pas perpétuer la « mentalité résolument optimiste » qui imprégnait profondément les programmes (apparents) de génération de revenus destinés aux femmes au cours des premières années : les qualifications et la formation professionnelle avaient été mises en place au profit des femmes pour « les occuper » mais n'étaient pas particulièrement lucratifs. Une approche privilégiant le sens des affaires est plus réaliste et est nettement plus prometteuse pour un succès à long terme.

- À moins que les filles n'acquièrent des compétences évolutives, flexibles, elles risquent de se prendre au piège dans un cycle qui leur est utile à court terme mais pas à long terme, lorsque la compétence qu'elles ont acquise ne sera plus d'actualité.

- La pérennité doit être assurée à long terme et doit aider les stagiaires à trouver du travail dans des secteurs économiques en rapide mutation et dans le contexte de la mondialisation. Une approche dans le cadre de l'entreprise, qui étudie soigneusement les marchés locaux et mondiaux, est essentielle. La recherche attentive des endroits où les opportunités existent, signifie qu'il ne faut pas avoir peur de s'engager dans le secteur des entreprises.

- L'un des aspects les plus rafraîchissants de l'approche basée sur les moyens de subsistance, sont relatif à son respect de la culture des jeunes ainsi que du conteste de leur vie. Les limites existantes de ce qui sont considérées comme activités appropriées pour les filles doivent être élargies de manière créative et réfléchie parce que les réseaux et la mobilité des filles diffèrent de ceux des garçons et à cause des différences fondées sur l'inégalité des sexes.

- Il faut avoir une meilleure connaissance des activités que les adolescentes souhaitent mener. Elles devraient recevoir les outils dont elles ont besoin pour réaliser leur potentiel et leurs aspirations - elles ont aussi besoin d'avoir de grandes ambitions.

- Les adolescentes travaillent parce qu'elles sont pauvres. À la lumière des niveaux élevés de pauvreté, une étude devrait porter sur la manière dont les réseaux peuvent être sensibles aux contraintes économiques et politiques auxquelles les jeunes font face, tout en faisant preuve de réalisme face à la manière dont les programmes sont exécutés au niveau des ONG. 


\section{QUE RÉSERVE L'AVENIR?}

Au cours des dernières années, les gouvernements, les ONG et les organisations des Nations Unies, les fondations, les groupes de recherche et une série d'autres partenaires, y compris le secteur privé, ont manifesté un intérêt de plus en plus marqué pour les jeunes. Il y a plusieurs raisons à cela, et on compte parmi celles-ci l'incidence croissante du VIH/SIDA et du tabagisme ; les problèmes sociaux comme la discrimination fondée sur le sexe et la violence et les problèmes de plus grande envergure comme l'impact de la mondialisation. Il apparaît maintenant clairement qu'un intérêt marqué pour les jeunes est important pour le présent et pour l'avenir — l'adolescence est une période de la vie qui fournit des opportunités de s'éloigner des cercles vicieux qui sapent le développement humain et les droits de la personne.

Avec l'attention croissante accordée aux jeunes, il est apparu un consensus de plus en plus large sur ce qui doit être fait pour garantir et protéger les droits des adolescents au développement, de même qu'une prise de conscience que les « solutions » sont communes à une série de problèmes interdépendants. Ces solutions comprennent le renforcement des capacités et des aptitudes physiques et psychosociales des adolescents, l'amélioration de leur accès à une gamme de services et d'opportunités, la création d'environnements sûrs et accueillants dans lesquels ils peuvent vivre et apprendre et l'assurance qu'ils sont en mesure de participer à la prise des décisions et des mesures qui affectent leur vie. Ces éléments sont décrits dans le document sur les Problèmes émergents élaboré pour la première Commission préparatoire de la session extraordinaire de l'Assemblée générale des Nations Unies sur les enfants y compris les adolescents, en 2001.

Le développement des capacités qui permettent aux adolescents de gagner leur vie et la création d'opportunités d'autosuffisance contribueront considérablement à garantir et à protéger leur droits au développement et à la santé. Les moyens de subsistance sont intrinsèquement importants car ils aident les adolescentes à atteindre des niveaux de vie décents, à accroître leurs choix et à avoir foi en l'avenir. Ils sont également importants parce qu'ils contribuent à renforcer les facteurs de protection (y compris, l'orientation, les structures et les opportunités) qui empêchent toute une série de comportements à risque et des situations qui minent la santé et le développement des adolescentes et qui les exposent à l'exploitation et aux abus.

Il est clair qu'il y a encore beaucoup à faire pour perfectionner et développer nos façons collectives de concevoir des politiques et programmes qui portent sur les moyens de subsistance des adolescentes 
et des adolescents. Il faut que le débat reste ouvert au fur et à mesure que la réflexion se développe dans ce domaine et il faut passer de l'intérêt porté à la formation professionnelle à une approche plus globale des moyens de subsistance; de la préoccupation relative à la protection des adolescents contre l'exploitation et les conditions de travail dangereuses, à un intérêt plus marqué pour les moyens de subsistance comme contribution positive à leur développement (et le développement de leur famille et de leur communauté) ; de la perception du travail comme un fardeau, à la perception des moyens de subsistance comme une opportunité ; d'une discussion « dichotomique » sur l'éducation et le travail à une méthode convergente de concevoir la programmation pour les adolescents.

Il faut poursuivre la promotion de ce domaine de programmation par le truchement d'une série d'arguments tirés de domaines comme l'économie politique, la santé publique et les droits de la personne. Cependant, il faut non seulement être capable d'avancer des arguments convaincants pour prendre des mesures (y compris les coûts sociaux et économiques de l'absence de moyens de subsistance pour les adolescentes), nous devons également définir plus clairement les domaines d'action prioritaires et prouver que ce qui doit être fait peut l'être durablement de manière raisonnable et à une certaine échelle acceptable. Il est probable qu'au cours des prochaines années, nous devrons beaucoup compter sur les ONG pour l'élaboration des projets pilotes dont nous aurons besoin pour convaincre les gouvernements et le secteur privé de consacrer des ressources aux moyens de subsistance des adolescents.

L'approche basée sur les moyens de subsistance renforce une grande partie des questions dont le secteur de l'éducation discute actuellement, comme la réduction des disparités et de l'exclusion d'une part, et l'amélioration de la qualité et de la pertinence de l'éducation d'autre part. Le Forum mondial sur l'éducation qui s'est déroulé en avril 2000 à Dakar, au Sénégal — et la réunion précédente, la Conférence mondiale sur l'éducation pour tous en 1990 — ont apporté une contribution importante à ce domaine de la programmation grâce à des approches formelles et alternatives pour la mise en place d'environnements d'apprentissage sûrs et accueillants au profit des adolescents. Les adolescents doivent être encouragés à développer toute une série de compétences comme les capacités de lecture et d'écriture et les capacités au calcul ; les connaissances pratiques (compétences psychosociales) ; les compétences spécialisées et les compétences en gestion d'entreprise, y compris les habiletés pratiques (par exemple comment accéder au crédit), les aptitudes sociales (par exemple comment travailler avec les autres) ainsi que les compétences en gestion et les aptitudes stratégiques (par exemple comment 
reconnaître les conséquences à long terme des choix actuels).

Au fur et à mesure que nous avancerons dans le domaine de la programmation, il sera important d'identifier les questions importantes. Par exemple, le VIH/SIDA présente un éventail d'opportunités en matière de contribution à la prévention et la lutte contre cette pandémie par l'intermédiaire de la prévention à long terme (le SIDA est de plus en plus une maladie de la pauvreté) et grâce à la prise de mesures à l'égard des adolescents affectés par le VIH/SIDA, y compris ceux d'entre eux devenus orphelins et qui sont chefs de ménage. Cela est aussi probablement le cas de la violence, eu égard à la prévention et aux mesures à prendre.

L'accent mis sur les moyens de subsistance renforcera davantage l'intérêt porté aux adolescents en tant que ressources et atouts à développer, plutôt que comme problèmes ou comme dépositaires des comportements à haut risque. Plusieurs adolescents contribuent déjà de manière significative à la vie de leur famille ou de leur communauté, y compris la satisfaction des besoins de survie et de développement de leurs frères et sœurs plus jeunes. Une approche basée sur les moyens de subsistance peut contribuer à créer des opportunités pour les adolescents ; assurer qu'ils tirent parti de leurs contributions ; et les empêcher de s'engager dans des emplois dangereux, dans lesquels ils sont l'objet d'exploitation et d'abus et qui violent leurs droits.
Il faut de plus en plus subdiviser la période de l'adolescence, avec un accent particulier sur l'âge et le sexe, mais il faut aussi inclure des questions comme la réussite scolaire et le statut matrimonial afin de s'assurer que l'on tire parti des nouvelles capacités des adolescents. De plus, il est important d'avoir une perception claire des différents besoins des adolescents (de 10 à 19 ans) et des jeunes (de 15 à 24 ans). Et alors que nous devons tirer les enseignements de la vaste expérience des programmes de subsistance, nous devons porter un regard critique sur l'application des bonnes pratiques aux adolescents : par exemple les enseignements tirés de l'expérience des jeunes plus âgés peut avoir une pertinence moins évidente pour ceux âgés de 15 à 18 ans.

À l'évidence, les moyens de subsistance nous offrent la possibilité de nous intéresser aux nombreux autres problèmes de société, par exemple les différents besoins des garçons et des filles et les besoins des plus défavorisés et des plus marginalisés. Il y a des questions majeures auxquelles il faut apporter des réponses dont un certain nombre sont traitées dans ce rapport bien que la majeure partie de ce qui doit être appris soit susceptible de l'être au cours de ce processus. Cela met en exergue l'importance de mettre en rapport les programmes de subsistance avec la recherche et de poursuivre la mise au point des types d'activités novatrices qui ont été présentées et ont fait l'objet d'une discussion au cours de l'atelier. 


\section{NOTES}

1 Le résumé ci-dessous est principalement basé sur la communication de Cynthia Lloyd.

2 Il serait instructif d'en savoir davantage sur l'expérience acquise par ceux qui ont effectué le service national ; savoir par exemple si le service est obligatoire ou s'il se fait sur la base du volontariat et si seuls les hommes y sont admis ou alors hommes et femmes également. Il est clair que le service national a des implications sur l'avenir, s'il est obligatoire pour les hommes et s'il permet d'acquérir des qualifications.

3 Ces données n'incluent pas les étudiants travailleurs, parce que leur activité première en qualité d'étudiant prend le pas sur leur statut de travailleur ; elles n'incluent pas non plus les nouveaux venus sur le marché du travail qui n'ont pas encore trouvé d'emploi, ni les enfants âgés de moins de 15 ans. En outre, le période de référence peut varier d'un pays à l'autre, de sorte que dans certains cas, quelqu'un qui a travaillé cinq jours ou plus peut être pris en compte, cependant que dans d'autres, une période beaucoup plus longue peut être exigée pour l'inclusion d'un travailleur.

4 Valerie Durrant a introduit le concept des « filles qui ne font rien » au cours de l'atelier. Elle a aussi présenté le cas du Pakistan.

5 Le travail à la maison a été considéré par ceux qui ont collecté les données comme un travail rémunéré. Par exemple les filles ont été interrogées sur le point de savoir si elles produisaient de la broderie pour la vente ou pour leur usage propre à la maison. Les filles considérées comme « ne faisant rien » ne travaillaient pas à la maison.
6 La section inclut des éléments relatifs aux communications de Sahar El-Tawila et Safa'a El-Kogali.

7 La récente sortie de l'Egypte d'une période d'ajustement structurel a eu un impact sur le marché de l'emploi et sur celui du mariage. L'un des effets ressentis dans certains secteurs y compris celui de la confection, est illustré par le fait que des personnes illettrées travaillent avec des employés qui ont un certain niveau d'éducation. Le fait que des filles illettrées au même titre que des filles alphabétisées, occupent des emplois non qualifiés dans le secteur de la confection indique que des facteurs autres que la pauvreté influencent leur entrée dans le marché de l'emploi. Les filles dont le niveau d'éducation est le moins élevé commencent à se percevoir comme les égales de celles qui sont plus éduquées parce qu'elles font le même travail pour les mêmes rémunérations.

8 Une communication a été présentée sur ce sujet par Simel Esim.

9 Ces questions ont été abordées par Simel Esim au cours de sa communication.

10 Alec Fyfe a présenté une communication sur ce sujet.

11 Cette partie s'inspire de la communication de Nagah Hassan sur les premières recherches qu'elle a menées sur ce sujet.

12 Cette partie s'inspire de la communication de Mary Kawar sur les premières recherches qu'elle a menées sur ce sujet.

13 La communication de Sajeda Amin est basée sur ses recherches au Bangladesh.

14 Le résumé est basé sur les communications de Simel Esim et Jamie Schnurr ainsi que sur leurs travaux à l'ICWR et au CRDI respectivement. 
15 Le résumé est basé sur les travaux de Jennefer Sebstad.

16 Cette section s'inspire des informations présentées par Joachim Victor Gomes.

17 Mohammad Ibrahim, directeur du CMES, a présenté une vue d'ensemble de ses travaux.

18 Sagri Singh a présenté des informations sur les moyens de subsistance des filles en Inde.
19 Cette section s'inspire de la communication présentée par Banu Khan, Annabel Erulkar et Stephen Mirero.

20 John Grierson, Harun Bhaiya et Najma Sharif ont présenté des communications sur les problèmes de formation. Cette section s'inspire de leurs travaux.

\section{RÉFÉRENCES}

Bureau international du travail (BIT). 1993, 1994. Year Book of Labour Statistics. Genève, Suisse : BIT.

Bureau international du travail (BIT). 1996. "Child labor: What is to be done?" document pour la discussion à l'Informal Tripartite Meeting at the Ministerial Level. Genève, Suisse : BIT.

Chambers, Robert et Gordon R. Conway. 1992. "Sustainable rural livelihoods: Practical concepts for the 21st century," discussion paper no. 296. Sussex, Royaume-Uni: Institute of Development Studies.

El-Tawila, Sahar, Barbara Ibrahim, Omayma El Gibaly, Fikrat El Sahn, Sunny Sallam, Susan M. Lee, Barbara Mensch, Hind Wassef, Sarah Bukhari et Osman Galal. 1999. Transitions to Adulthood: A National Survey of Egyptian Adolescents. Le Caire, Égypte: Population Council.
Espínola, Basílica et al. 1988. In the Streets: Working Street Children in Asunción: A Book for Action. Colombie: Gente Nueva.

Mensch, Barbara, Judith Bruce et Margaret E. Greene. 1998. The Uncharted Passage: Girls' Adolescence in the Developing World. New York: Population Council.

Szanton Blanc, Cristina. 1994. Urban Children in Distress: Global Predicaments and Innovative Strategies. Langhorne, Pennsylvania: Gordon \& Breach Science Publishers, UNICEF. 


\section{A N N E X A L'ORDRE DU JOUR DE L'ATELIER}

Mercredi, le 13 octobre

\section{Bienvenu}

Geeta Rao Gupta, Barbara Ibrahim, Ellen

Marshall, Bruce Dick et Judith Bruce

\section{Qu'est-ce que c'est que l'approche} fondée sur les moyens de subsistance?

Présidé par Judith Bruce

- Vue d'ensemble (30 minutes) Simel Esim

- Un exemple de l'Afrique : la rationale et les opérations actuelles du Livelihood Network (Réseau de moyens de subsistance) de l'International Development Research Centre (10 minutes) Jamie Schnurr

- Discussion (20 minutes)

\section{Ou travaillent les adolescents?}

Présidé par Aboubacry Tall

- Données sur les expériences au travail des adolescents dans les pays en développement (20 minutes) Cynthia Lloyd

- La section égyptienne pour étudier à profondeur l'expérience du travail chez les filles et les garçons adolescents (20 minutes) - Safa'a El-Kogali

- Les méthodes pour apprendre et les résultats d'une investigation du mystère de la proportion élevée de filles en Pakistan qui ne sont pas mariées, ne travaillent pas et ne sont pas à l'école (20 minutes) — Valerie Durrant

- Discussion (1 heure)

\section{Le travail des filles et l'environnement politique et normatif}

Présidé par Barbara Ibrahim

- Vue d'ensemble des mesures de protection des enfants et les moyens de subsistance des adolescents, les standards internationaux du Bureau international de travail et vue d'ensemble de la stratégie du Programme international pour l'élimination du travail chez les enfants (Theresa Smout) et les applications au niveau du pays (Alec Fyfe) (30 minutes)

- Le lien entre le travail et l'éducation passé en revue : la litérature disponible et des interpretations alternatives des avantages/désavantages du travail et de l'école (Simel Esim) et la perspective égyptienne (Sahar El-Tawila) (20 minutes)

- Discussion (40 minutes)

\section{Comment les jeunes adolescentes perçoivent-elles leurs conditions de travail ?}

Présidé par Sajeda Amin

- Perspectives de trois pays sur leurs estimations des opinions des adolescentes à propos de leur travail et les opportunités offertes (2 heures)

- Recherche sur les travailleuses dans le secteur de la confection au Bangladesh — Sajeda Amin

- Pourquoi les jeunes femmes en Jordanie travaillent ; les attitudes de leurs familles et comment ces attitudes affectent les opportunités de travail ; et l'attitude des adolescentes sur leurs propres expériences - Mary Kawar

- Études de cas de jeunes femmes travailleuses en Égypte - Nagah Hassan 
Jeudi, le 14 octobre

\section{Quelle est l'expérience jusqu'au}

présent des programmes à l'appui ou

à la génération des moyens de subsis-

\section{tance pour les adolescentes?}

Présidé par Sharon R. Lapp

- Introduction pour réiterer la définition et le cadre théorique des moyens de subsistance et pour ébaucher des domaines possibles pour l'action (15 minutes) Jennefer Sebstad

- Stratégies pour le microfinance

- Vue d'ensemble des stratégies de microfinance, y compris des programmes qui offrent une fenêtre aux adolescentes qui s'orientent vers les marchés soutenables, d'échelle et d'approfondissement ainsi que des programmes où les services financières font partie d'une politique catégorique de développement social (15 minutes) - Jennefer Sebstad

- Résumé des résultats d'une revue de programmes à l'étendu des adolescents par les institutions bangladaises de microfinance (15 minutes $+5-10$ minutes de discussion) Joachim Victor Gomes

- L'expérience du Kenya Rural Enterprise Program comme exemple d'une institution populaire de microfinance qui travaille avec des adolescentes en tant que politique catégorique et expérimentation programmatique (20 minutes +10 minutes de discussion) Banu Khan, Annabel Erulkar et Stephen Mirero

- Résultats d'interviews avec des institutions indiennes de microfinance (20 minutes +10 minutes de discussion) - Sagri Singh
- D’autres innovations financières

- L'expérience du Centre for Mass Education in Science en offrant le crédit à 3000 adolescentes dans le contexte d'une schéma de développement social (15 minutes) Mohammad Ibrahim

- Une enquête sur le meilleur moyen d'offrir des opportunités d'épargne pour les travailleuses de confection (15 minutes) - Joachim Victor Gomes

- Discussion (30 minutes)

- L'entrainement pour le développement entrepreneuriel

Présidé par Simel Esim

- Comment l'entrainement pour le développement entrepreneuriel peut s'adresser aux besoins des adolescents pour les moyens de subsistance (John Grierson) et commentaires (Harun Bhaiya et Najma Sharif) (45 minutes)

- Discussion (30 minutes)

- Réflections sur l'expérience de l'UNICEF Présidé par Bruce Dick

- Discussion de la connaissance accrue en cherchant le contexte de moyens de subsistance au milieu du développement et des droits ; la séparation entre l'empêchement du travail des enfants et l'encouragement des moyens de subsistance chez les adolescents ; et les expériences des pays.

\section{Conclusion}

Judith Bruce, Geeta Rao Gupta, Barbara

Ibrahim et Jennefer Sebstad 


\section{A N N EXE B LISTE DE PARTICIPANTS DE L'ATELIER}

\author{
Romany Abadir \\ Centre for Development and Population Activities \\ 53, Manial Street, Suite 500 \\ Manial El Rodah \\ P.O. Box 110 \\ Le Cairé 11451, Égypte \\ téléphone: $20-2-365-4567$ \\ fax: 20-2-365-4568 \\ e-mail (group): cedpa@intouch.com
}

Rahnuma Afrin, Officier du programme ActionAid Bangladesh

House \#41, Road \#8

Dhanmondi R/A, Dhaka 1205, Bangladesh

téléphone: 880-2-811-802/763, 810-933

fax: 880-2-813-150

e-mail: aabcr@dhaka.agni.com

Sajeda Amin, Associée

Population Council

One Dag Hammarskjold Plaza

New York, NY 10017

téléphone: 212-339-0680

fax: 212-755-6052

e-mail:samin@popcouncil.org

Dang Nguyen Anh

Institute of Sociology

27 Tran Xuan Soan Street

Hanoi, Viet Nam

téléphone: 84-4-733-0577

fax: 84-4-733-0588

e-mail: pc_anh@netnam.org.vn

\section{Marie Assaad}

1095 Corniche El-Nil

3rd floor, Garden City

Le Caire, Égypte

téléphone: 20-2-794-3305

Paul Bennell, Consultant

60 Rugby Road

Brighton, East Sussex

BN1 6ED, Royaume-Uni

téléphone: 44-1-273-503259

e-mail: swainson@bennell.u-net.com
Harun Bhaiya, Executif en chef

Strengthening Informal Sector Training and

Enterprise

MultiChoice Towers, 5th floor, Lower Hill Road

P.O. Box 34336

Nairobi, Kenya

téléphone: 254-2-718-155/716-099

fax: 254-2-716-059

e-mail: hbhaiya@africaonline.co.ke

Leila Bisharat, Representative

United Nations Children's Fund

87 Misr Helwan Agriculture Road

Maadi, Le Caire, Égypte

téléphone: 20-2-526-5083

fax: 20-2-526-4218

e-mail: Ibisharat@unicef.org

Martha Brady, Associée de programme

Population Council

One Dag Hammarskjold Plaza

New York, NY 10017

téléphone: 212-339-0618

fax: 212-755-6052

e-mail:mbrady@popcouncil.org

Carlos Brambila-Paz, Associé de programme, Frontiers

Population Council

Adresse du courrier :

P.O. Box 593478

Miami, FL 33159-3478

Situation du bureau:

8a Calle 14-44, Zona 13

Guatemala City 01013, Guatemala

téléphone: 502-3-32-3848/2365

fax: 502-3-62-7227

e-mail: cbrambila@guate.net

Judith Bruce, Directrice de programme,

Gender, Family, and Development

Population Council

One Dag Hammarskjold Plaza

New York, NY 10017

téléphone: 212-339-0641

fax: 212-755-6052

e-mail: jbruce@popcouncil.org 
Bonnie Brusky, Officier de programme South Asian Network of Microfinance Initiatives House 206, Road 10 New DOHS, Mohakhali Dhaka 1206, Bangladesh téléphone: 880-2-887-200 e-mail: bjbrusky@club-internet.fr; bjbrusky@mailexcite.com

Bruce Dick, Conseiller supérieur, Youth Health, Programme Division United Nations Children's Fund 633 Third Avenue, 24th floor New York, NY 10017 téléphone: 212-824-6324

fax: 212-824-6465

e-mail: bdick@unicef.org

\section{Valerie Durrant}

National Research Council

Committee on Population

2101 Constitution Avenue NW

Washington, DC 20418

téléphone: 202-334-3167

fax: 202-334-3768

e-mail:vdurrant@nas.edu

Willem van Eekelen, Expert associé,

Politiques du marché de travail

International Labour Organization, Cairo

9 Taha Hussein Street

Zamalek, Le Caire, Égypte

téléphone: 20-2-341-9290/340-0123

fax: 20-2-341-0889

e-mail: eekelen@ilo.org

Safa’a El-Kogali, Économiste

The World Bank

1818 H Street NW

Washington, DC 20433

téléphone: 202-458-9869

e-mail: selkogali@worldbank.org

Sahar El-Tawila, Associée de recherche Social Research Center

American University in Cairo

Le Caire, Égypte

téléphone: 20-2-357-6947

fax: 20-2-355-7298

e-mail: sahart@auc.aucegypt.edu

Annabel Erulkar, Personnel associé de programme Population Council

General Accident House

Ralph Bunche Road

Nairobi, Kenya

téléphone: 254-2-713-480/1/2/3

fax: 254-2-713-479

e-mail: aerulkar@popcouncil.or.ke
Simel Esim, Economiste

International Center for Research on Women

1717 Massachusetts Avenue NW, \#302

Washington, DC 20036

téléphone: 202-797-0007

fax: 202-797-0020

e-mail: Simel_Esim@dai.com

\section{Tamara Fetters}

Ipas USA

300 Market Street, Suite 200

Chapel Hill, NC 27516

téléphone: 919-967-7052

fax: 919-929-0258

e-mail: fetters@ipas.org

Alec Fyfe, Conseiller supérieur, Travail des enfants United Nations Children's Fund

3 United Nations Plaza, T26A

New York, NY 10017

téléphone: 212-824-6639

fax: 212-824-6485

e-mail: afyfe@unicef.org

Joachim Victor Gomes, Consultant indépendent 147-E Green Road, Flat E-3

Dhaka 1205, Bangladesh

e-mail:snmjvg@bdonline.com; vicg32@yahoo.com

John Grierson, Spécialiste supérieur,

Développement d'entreprises

FTP International Ltd.

P.O. Box 484

FIN-00101 Helsinki, Finlande

téléphone: 358-9-7701-3323

fax: 358-9-7701-3498/3499

e-mail: john.grierson@ftpinter.fi

Geeta Rao Gupta, Présidente

International Center for Research on Women

1717 Massachusetts Avenue NW, \#302

Washington, DC 20036

téléphone: 202-797-0007

fax: 202-797-0020

e-mail: geeta@icrw.org

Nicole Haberland, Associée de programme

Population Council

One Dag Hammarskjold Plaza

New York, NY 10017

téléphone: 212-339-0676

fax: 212-755-6052

e-mail: nhaberland@popcouncil.org 
Minhaj ul Haque, Officier de programme Population Council

House No. 7, Street No. 62

Sector $\mathrm{F} 6 / 3$

Islamabad, Pakistan

téléphone: 92-51-277-439

fax: 92-51-821-401

e-mail:minhaj@pcpak.org

Nagah Hassan, Assistante de recherche Population Council

P.O. Box 115

Dokki 12211

Le Caire, Égypté

téléphone: 20-2-573-8277

fax: 20-2-570-1804

e-mail: nhassan@pccairo.org

Karen Heissler, Officier assistant de programme, Section protection

United Nations Children's Fund

P.O. Box 38

Dhaka-1000, Bangladesh

téléphone: 880-2-933-6701/2/3/ . . /20

fax: 880-2-933-5641/2

e-mail: kheissler@mail.unicef.bangla.net

Aziza Helmy, Officier de femmes

en développement

United States Agency for International

Development

Mansour Building

Zahraa El Maadi

Le Caire, Égypte

téléphone: 20-2-516-5505

fax: 20-2-521-8501

e-mail: ahelmy@usaid.gov

\section{Ishrat Husain}

USAID/Bureau africain (AFR/SD)

1325 G Street NW, Suite 400

Washington, DC 20005

téléphone: 202-219-0477

fax: 202-216-3373

e-mail: ihusain@afr-sd.org

Barbara Ibrahim, Directrice régionale, Asie de l'ouest et Afrique du nord

Population Council

P.O. Box 115

Dokki 12211

Le Caire, Égypte

téléphone: 20-2-573-8277

fax: 20-2-570-1804

e-mail: bibrahim@pccairo.org
Mohammad Ibrahim, Directeur executif

Centre for Mass Education and Science

828 Dhanmondi Road \#19

Dhaka 1209, Bangladesh

téléphone: 880-2-811-898

fax: 880-2-803-559

e-mail: ibrahim@citechco.net; cmes@citechco.net

Saad el Din Ibrahim, Directeur

Ibn Khaldoun Center for Development Studies

17, Street 12 Maqattam

P.O. Box 13

Maqattam, Le Caire, Égypte

téléphone: 20-2-508-1617

fax: 20-2-508-1030

e-mail: semibrahim@hotmail.com

Lubna Izziddin, Assistante de programme, Projet adolescent

United Nations Children's Fund, Jordanie

P.O. Box 1551

Amman 111821, Jordanie

téléphone: 962-6-553-9977

fax: 962-6-553-1112

e-mail: Lubna_Izziddin_at_P0320A01@

smtplink.unicef.org

\section{Laila Kamel}

11 Gabalaya Street

Apt. \#9, 3è étage

Zamalek, Le Caire, Égypte

téléphone: 20-2-332-0752/0832

fax: 20-2-340-2660

e-mail: cid@intouch.com

\section{Mary Kawar}

Aresco Centre

P.0. Box 11-4088

Beyrouth, Liban

téléphone: 961-1-752-400, ext. 229

fax: 961-1-752-406

e-mail: kawar@ilo.org.lb

Amira Kazem, Economiste

Secteur développement humain

The World Bank

World Trade Center

1191 Corniche El-Nil, 15th floor

Le Cair, Égypte

téléphone: 20-2-574-1670/1

fax: 20-2-574-1676

e-mail: akazem@worldbank.org 


\section{Banu Khan}

Population Council

General Accident House

Ralph Bunche Road

Nairobi, Kenya

téléphone: 254-2-713-480/1/2/3

fax: 254-2-713-479

e-mail: bkhan@popcouncil.or.ke

\section{Tamer Kirolas}

The Egyptian Small \& Micro-enterprise Association No. 2 Maroof Street, 4è étage

Le Caire, Égypte

téléphone: 20-2-577-7273/5

e-mail:esma@intouch.com

Jayasankar Krishnamurty, Economiste supérieur Politiques du travail et du marché de travail Departement de travail et d'entraînement International Labour Office

4 , route des Morillons

CH-1211 Genève 22, Suisse

téléphone: 41-22-799-8946

fax: 41-22-799-7678

e-mail: krishnamurty@ilo.org; sunanda9@yahoo.com

Attallah Kuttab, Directeur

Save the Children

P.O. Box 580

Ataba, Le Caire, Égypte

téléphone: 20-2-353-4505

fax: 20-2-355-6343

e-mail: careegp@starnet.com.eg

Sharon R. Lapp, Officier de programme The Ford Foundation

P.O. Box 2344

Le Caire, Égypte

téléphone: 20-2-355-2121

fax: 20-2-355-4018

e-mail: s.lapp@fordfound.org

Susan M. Lee, Consultante indépendente 1724 V Street NW

Washington, DC 20009

téléphone: 202-518-5986

e-mail: susanmlee@juno.com

\section{Cynthia Lloyd}

Population Council

One Dag Hammarskjold Plaza

New York, NY 10017

téléphone: 212-339-0664

fax: 212-755-6052

e-mail: clloyd@popcouncil.org

\section{Ellen Marshall}

United Nations Foundation

1301 Connecticut Avenue NW, Suite 700

Washington, DC 20036

téléphone: 202-887-9040

fax: 202-887-9021

e-mail: emarshall@unfoundation.org

Krista Masonis, Consultante en développement c/o DPA/Karim el Gawhary

P.O. Box 366

Mohammed Farid, Maspero

Le Caire, Égypte

téléphone/fax: 20-2-508-7728

e-mail: kritsam@eis.egnet.net

\section{Pamela McCloud}

Centre for Development and Population Activities 53, Manial Street, Suite 500

Manial El Rodah

P.O. Box 110

Le Caire 11451, Égypte

téléphone: 20-2-365-4567

fax: 20-2-365-4568

e-mail (group): cedpa@intouch.com

Carey Meyers, Coordinatrice de programmes

Population Council

One Dag Hammarskjold Plaza

New York, NY 10017

téléphone: 212-339-0637

fax: 212-755-6052

e-mail: cmeyers@popcouncil.org

\section{Stephen Mirero}

Kenya Rural Enterprise Program

Chef exécutif, projets spéciaux

K-Rep Group Coordination Office

P.O. Box 39312

Nairobi, Kenya

téléphone: 254-2-572-422

fax: 254-2-711-645

e-mail (group): krep@arcc.or.ke

Richard Mkandawire, Directeur

Center for Youth Studies

Université de Venda

Private Bag X5050

Thohoyandou 0950, Afrique du Sud

téléphone: 27-159-824-757

fax: 27-159-824-756

e-mail: mkanda@mweb.co.za; mkandr@ caddy.univen.ac.za 
Nadir A. L. Mohammed, Economiste de pays, MNSED \& MNCEG, région moyen-orient et

Afrique du nord

The World Bank

World Trade Center

1191 Corniche El-Nil, 15th floor

Le Caire, Égypte

téléphone: 20-2-574-1670/1

fax: 20-2-574-1676

e-mail: nmohammed@worldbank.org

Valerie Moulay-Omar, Coordinatrice régionale d'opérations

Population Council

128 Sotrac Mermoz

P.O. Box 21027

Dakar, Sénégal

téléphone: 221-824-1993/4

fax: 221-824-1998

e-mail: vmo@telecomplus.sn

Eglal Rached, Directeur régional

Moyen orient et Afrique du nord

International Development Research Centre

3 Amman Square, 5th floor

Dokki, Le Caire, Égypte

téléphone: 20-2-336-7051/2/3

fax: 20-2-336-7056

e-mail: erached@idrc.org.eg

Eva Rathgeber, Directrice régionale

Afrique de l'est et australe

International Development Research Centre

P.O. Box 62084

Nairobi, Kenya

téléphone: 254-2-713-160/1

fax: 254-2-711-063

e-mail: erathgeber@idrc.or.ke;

EvaRathgeber@netscape.net

Jane Rosser, Conseillère supérieure de programme, éducation du monde

27 Myrtle Street

Brattleboro, VT 05301

téléphone: 802-254-3893

e-mail: rossvita@sover.net

Lydia Zoungrana-Saloucou, Experte en recherche

Population Council

01 BP 6250, Cité An III

Ouagadougou 01, Burkina Faso

téléphone: $226-31-1242 / 3$

fax: 226-31-1246

e-mail: Isaloucou@popcouncil.bf
Zeba Sathar, Associée de programme

Population Council

House No. 7, Street No. 62

Sector $\mathrm{F} 6 / 3$

Islamabad, Pakistan

téléphone: 92-51-277-439

fax: 92-51-821-401

e-mail: zsathar@pcpak.org

Jamie Schnurr, Specialiste en recherche,

Moyens de subsistance des adolescents

International Development Research Centre

P.O. Box 8500

Ottawa, ON

Canada KIG 3 H9

téléphone: 613-236-6163, ext. 2320

fax: 613-567-7748

e-mail: jschnurr@idrc.ca

Jennefer Sebstad, Consultante indépendente

P.O. Box 18973

Addis Ababa, Ethiopie

Courrier aux États-Unis :

c/o HIID

14 Story Street

Cambridge, MA 02138

téléphone: 251-1-712-626

e-mail: jsebstad@telecom.net.et;

jsebstad@aol.com

Najma Sharif, Professeur en économie

St. Mary's University

6 Village Crescent

Bedford, Nouvelle-Écosse

Canada B4A 1J2

téléphone: 902-420-5616

e-mail: najma.sharif@stmarys.ca

Andrew Simmons, Officier de programme en chef Commonwealth Youth Program

Gender and Youth Affairs Division

Commonwealth Secretariat

Marlborough House, Pall Mall

Londres SWIY 5HX, Royaume-Uni

téléphone: 44-20-7839-3411

fax: 44-20-7930-1647

e-mail: simmonsa@commonwealth.int

Marja Simojoki, Officier de programme

Ambassade de Finlande, Département de coopération en développement

P.O. Box 30379

Nairobi, Kenya

téléphone: 254-2-336-717/740, 334-408

fax: 254-2-335-986

e-mail: finland@form-net.com 


\section{Sagri Singh}

2850 North Charles Street, \#14C

Baltimore, MD 21218

téléphone: 410-662-7025

e-mail: emailsagri@yahoo.com

Allison Smith, Consultante, Adolescent Reproductive Health

P.O. Box 1573

Red Lodge, MT 59068

e-mail: smitha@hsph.harvard.edu

\section{Theresa Smout}

Gender Focal Point

International Program on the Elimination of Child Labour

International Labour Organization

4 , route des Morillons

CH-1211 Genève 22, Suisse

téléphone: 41-22-799-6929

fax: 41-22-799-8771

e-mail:smout@ilo.org

Aboubacry Tall, Senior Programme Officer United Nations Children's Fund, Egypt

87 Misr Helwan

Agricultural Road

Maadi, Le Caire, Égypte

téléphone: 20-2-526-5083/4/5/6/7

fax: 20-2-526-4218

e-mail: atall@unicef.org
Eve Thompson, Directrice de pays

Joint Center for Political and Economic Studies 20 Melle Street, 4th floor, Van der Stel Place

Braamfontein, 2017

P.O. Box 23881

Joubert Park 2044, Afrique du Sud

téléphone: 27-11-403-8641

fax: 27-11-339-8386

e-mail: jcsa@wn.apc.org

\section{Hind Wassef}

10 El-Saleh Ayoub Street

Apt. 24, 2nd floor

Zamalek, Le Caire, Égypte

téléphone: 20-2-217-7454

e-mail: hwassefh@yahoo.co.uk

\section{Salma Youssef}

Economic Research Forum

7 Bolous Hanna

Dokki, Égypte

téléphone: 20-2-337-0810

fax: 20-2-348-5553

e-mail: erf@idsc.gov.eg

Nadia Zibani, Chercheuse

16 Rue Vladimir Komorov

93200 Saint Denis, France

e-mail: nadiazibani@hotmail.com 


\section{A Population Council}

One Dag Hammarskjold Plaza

New York, New York 10017

téléphone: 001 212-339-0500

fax: 001 212-755-6052

e-mail: pubinfo@popcouncil.org

www.popcouncil.org
||||$R W$

1717 Massachusetts Avenue NW, Suite 302

Washington, DC 20036

téléphone: 001 202-797-0007

fax: 001 202-797-0020

e-mail: info@icrw.org

www.icrw.org 